\title{
A LINK TO THE PAST: USING MARKOV CHAIN MONTE CARLO FITTING TO CONSTRAIN FUNDAMENTAL PARAMETERS OF HIGH-REDSHIFT GALAXIES
}

\author{
N. Pirzkal ${ }^{1}$, B. Rothberg ${ }^{1,2}$, Kim K. Nilsson ${ }^{3}$, S. Finkelstein ${ }^{4}$, Anton Koekemoer $^{1}$, \\ SAngeEta Malhotra ${ }^{5}$, And James RhoAds ${ }^{5}$ \\ ${ }^{1}$ Space Telescope Science Institute, 3700 San Martin Drive, Baltimore, MD 21218, USA; npirzkal@ stsci.edu \\ ${ }^{2}$ George Mason University, Department of Physics \& Astronomy, MS 3F3, 4400 University Drive, Fairfax, VA 22030, USA \\ ${ }^{3}$ ST-ECF, Karl-Schwarzschild-Straße 2, 85748 Garching bei München, Germany \\ ${ }^{4}$ George P. and Cynthia Woods Mitchell Institute for Fundamental Physics \& Astronomy, Department of Physics and Astronomy, \\ Texas A\&M University, College Station, TX 77843, USA \\ ${ }^{5}$ School of Earth \& Space Exploration, Arizona State University, P.O. Box 871404, Tempe, AZ 85287-1404, USA \\ Received 2011 March 29; accepted 2012 January 29; published 2012 March 14
}

\begin{abstract}
We have developed a new method for fitting spectral energy distributions (SEDs) to identify and constrain the physical properties of high-redshift $(4<z<8)$ galaxies. Our approach uses an implementation of Bayesian based Markov Chain Monte Carlo that we have dubbed " $\pi \mathrm{MC}^{2}$." It allows us to compare observations to arbitrarily complex models and to compute $95 \%$ credible intervals that provide robust constraints for the model parameters. The work is presented in two sections. In the first, we test $\pi \mathrm{MC}^{2}$ using simulated SEDs to not only confirm the recovery of the known inputs but to assess the limitations of the method and identify potential hazards of SED fitting when applied specifically to high-redshift $(z>4)$ galaxies. In the second part of the paper we apply $\pi \mathrm{MC}^{2}$ to thirtythree $4<z<8$ objects, including the spectroscopically confirmed Grism ACS Program for Extragalactic Science Ly $\alpha$ sample $(4<z<6)$, supplemented by newly obtained Hubble Space Telescope/WFC3 near-IR observations, and several recently reported broadband selected $z>6$ galaxies. Using $\pi \mathrm{MC}^{2}$, we are able to constrain the stellar mass of these objects and in some cases their stellar age and find no evidence that any of these sources formed at a redshift larger than $z=8$, a time when the universe was $\approx 0.6$ Gyr old.
\end{abstract}

Key words: galaxies: evolution - galaxies: fundamental parameters - galaxies: high-redshift - methods: statistical

\section{INTRODUCTION}

Without a doubt, high-redshift galaxies are critical to developing a comprehensive picture of galaxy evolution. It is no longer sufficient to simply detect and catalog their existence. The next step is to determine fundamental parameters such as mass, metallicity, extinction, and the ages of their stellar population(s). Such archaeological reconstructions are by no means a simple feat and present significant technical challenges not just for the observations but also for the methods used to deduce their properties. In the local universe, it is relatively straightforward to determine mass from kinematic observations (i.e., velocity dispersions, rotational velocity) or information about the stellar populations from stellar absorption lines and nebular emission lines. However, at high- $z$, such observations are impractical, if not impossible with current technology. This forces us to rely on broadband photometry or deep lowresolution spectroscopy of bright emission lines (if we are particularly fortunate) to derive fundamental parameters. One popular technique to glean such information is fitting templates of stellar populations, computed from either theoretical isochrones or empirical observations, to the spectral energy distribution (SED) of distant galaxies. The galaxy SEDs are simply broadband photometric measurements obtained in as many filters as possible. Ideally, SED fitting should allow one to estimate the total stellar mass of the galaxy, the age and metallicity of the stellar population, and the amount of extinction in galaxies at known redshifts. However, in most cases, the redshifts of the observed galaxies are themselves unknown, adding it to the list of parameters to be determined. The total stellar mass of the galaxy is derived from determining the best possible scale factor to fit the stellar population template to the observations. In the simplest case of SED fitting, stellar templates of fixed metallic- ity are used, along with specific extinction laws (e.g., Calzetti, foreground dust, mixed dust, and stars), making the minimum number of parameters to fit only four (age, $A_{\mathrm{V}}$, mass, and $z$ ). In recent years, many have attempted to fit two stellar populations to the SED, which increases the number of free parameters to seven (Pirzkal et al. 2007; Nilsson et al. 2011). Complicating things further, metallicity is often left as another free parameter. This gives us five free parameters for single-population fits and nine free parameters for two-population fits! Moreover, many of these parameters are degenerate, that is, the effect on the SED colors can be the same for two or more different parameters. The implication of this is that, unless some assumptions are made about the values of at least a few of the parameters (i.e., independent redshift determination), the size of the parameter space to examine is daunting.

Undeterred from the seemingly large parameter space to explore, the popularity and frequency of SED fitting has increased significantly in the last few years (e.g., Mobasher et al. 2005; Pirzkal et al. 2007; McLure et al. 2009; Labbé et al. 2010) due to the availability of large catalogs of candidate galaxies obtained from deep surveys. Templates from Bruzual \& Charlot (2003, hereafter BC03), Maraston (2005, hereafter M05), or the newer, but unpublished S. Charlot \& G. Bruzual (2012, in preparation, hereafter CB11) are the most commonly used for comparisons. Bruzual \& Charlot (2003) rely heavily on theoretical isochrones, while Maraston (2005) and S. Charlot \& G. Bruzual (2012, in preparation) include empirical spectral data (primarily for red supergiants and asymptotic branch stars). Where the SED fitting methods differ is in how they span the rather extensive parameter space of input template parameters and how they derive errors for their results. One way to reduce the voluminous parameter space is to compute models only over a finite grid of input parameters, where each point on the grid is a 
distinct template model SED. However, this grid must be carefully selected and deriving error bars for each of the parameter of the best-fitting model is difficult and time consuming. Furthermore, using a preset grid of model parameters introduces a problem similar to selecting an appropriate number of bins for histograms. In this case, it is the input parameter grid that must be pre-selected and the actual choice of parameter values can affect the outcome of the fit. Ideally, a very fine grid should be used for each parameter, but in practice the size and span of the parameter grid is kept small enough, and sparse enough, to make the computational time manageable. There are several dangers that can result from this method, such as inefficiency, excluding ranges in parameter space that ultimately may prove the most realistic, and simply selecting the "best-fit" model from a coarsely sampled parameter space. In some cases, this can produce seemingly implausible results (i.e., a very old object in a young universe as is shown in Labbé et al. 2010; Richard et al. 2011).

A novel way to circumvent these limitations is to use Markov Chain Monte Carlo (MCMC) methods. As will be described in more detail later in the text, the MCMC method allows for an efficient and full exploration of parameter space in a reasonable amount of time and computing power. When based on maximum likelihood statistics, MCMC also allows for arbitrary complex models to be applied. In this paper, we have implemented an SED "fitting" method that relies on the principle of importance sampling, similar to what is done in numerical integration of complex numerical systems, but using an MCMC approach. In this sense, this method is not really a fitting method and should rather be regarded as a method to exclude or include ranges of model parameters. Unlike nearly all other SED fitting techniques which are affected by the degeneracy between model parameters, our method derives independent and separate posterior probability distributions for each of the model parameters. This allows for easy identification of the marginalization of some parameters, and more importantly, allows us to attach error bars to each of the physical characteristics derived for our sources. Using MCMC, marginalization does not require a priori knowledge of the parameter probability density functions, nor does it require a complicated multidimensional integral. MCMC has been used in the past (Sajina et al. 2006; Nilsson et al. 2007; Serra et al. 2011) and is becoming increasingly popular (Nilsson et al. 2011; Acquaviva et al. 2011). A comprehensive review of MCMC can be found in Trotta (2008).

As newer and more sensitive instruments probe longer wavelengths, making the high- $z$ universe more accessible, the number of potential galaxy sources has increased significantly, particularly in the last decade. The goal of this paper is to describe our MCMC approach to SED fitting based on modern computational techniques and demonstrate the insights we gain over more classical approaches using simulated galaxies and real observations. We start by describing our MCMC-based methodology in Section 2. We then test the effect of various models, the sizes of error bars, and photometric noise in Section 3. In Section 4, we use the MCMC approach to determine the physical properties of a sample of high-redshift galaxies: (1) the high-redshift $\operatorname{Ly} \alpha$ emitters $(4<z<6)$ first spectroscopically identified as part of the Grism ACS Program for Extragalactic Science (GRAPES) projects (Pirzkal et al. 2004, 2007) including several sources re-observed using the new WFC3 on Hubble Space Telescope (HST); (2) galaxy candidates at $z>7-8$ from Labbé et al. (2010); and (3) high-redshift lensed candidates from Bradley et al. (2008), Zheng et al. (2009), and Richard et al. (2011). In Section 5, we summarize our key findings and discuss future work to both improve our techniques and future observations.

All data and calculations in this paper assume $H_{\circ}=$ $70 \mathrm{~km} \mathrm{~s}^{-1} \mathrm{Mpc}^{-1}$ and a cosmology of $\Omega_{\mathrm{M}}=0.3, \Omega_{\lambda}=0.7$ $\left(q_{\circ}=-0.55\right)$. All flux measurements used and provided in Table 1 are in AB magnitudes.

\section{METHODOLOGY OF THE MCMC APPROACH}

\subsection{Description of the Markov Chain Monte Carlo Method}

MCMC is a random sampling method whereby the entire model parameter space is explored. It differs from traditional Monte Carlo methods in that it does not attempt to sample a multidimensional region uniformly. It instead aims at visiting a point $\mathbf{x}$ with a probability proportional to some given probability distribution function $\pi(\mathbf{x})$. The method will spend twice the time sampling a given subregion than a region that is half as likely. Given a set of observed data, D, all that is required is the ability to derive the likelihood of that data, given the model parameters $\mathbf{x}$. In return, one directly recovers the normalized probability density function of each input model parameter by observing how often the method sampled a volume $d \mathbf{x}$. The advantage of the MCMC approach is that the distribution of a single component $\mathbf{x}$ can be examined by marginalizing the other components. The posterior probability function (PDF) can be constructed by simply creating a histogram of the values of a given parameter in the MCMC chain, including only values taken after the chain was observed to converge. We were motivated to implement our new SED fitting technique because current methods that rely on reporting the best-fitting model often fail to fully explore the model parameter space. They also fail to fully capture the degeneracy between various model parameters (e.g., Labbé et al. 2010). Once the number of model parameters exceeds three or four, it rapidly becomes inefficient to fit SEDs using parameter grid based fitting methods. This is especially true when errors for each of the fitted parameters are estimated by repeating the grid-based fitting following a pure Monte Carlo approach (e.g., each parameter requires several thousand additional iterations just to obtain errors). Our main motivation for implementing our own MCMC fitting was to be able to consider complex input models, including the effect of nebular escape fraction $\left(f_{\text {esc }}\right)$, or models consisting of more than one stellar population (as was attempted in Pirzkal et al. 2007). Nebular emission lines and nebular continuum emission was shown in recent work that they can contribute greatly to young stellar populations (Zackrisson et al. 2008; Schaerer \& de Barros 2009; Raiter et al. 2010). It seems natural to apply the statistically sound method of MCMC to the world of astronomical SED fitting, especially when some insight can be gained from deriving proper estimates of model parameter credible intervals. When used properly, MCMC is a very powerful method to determine range in model parameter values that are consistent with the data down to a chosen confidence level. This confidence level is commonly taken to be at least $95.45 \%$ (sometimes described as $2 \sigma$ ), although there appear to be a trend to only report results with $68 \%(1 \sigma)$ confidence. It is our opinion that $68 \%$ credible regions are not constraining enough. In our experience, the posterior probability density we derive for model parameters is non-Gaussian and hence the $95 \%$ credible regions are not simply twice as wide as the $68 \%$ ones. While cumbersome, we have opted to sometimes quote and plot 
Table 1

Photometry

\begin{tabular}{|c|c|c|c|c|c|c|c|c|c|c|}
\hline UID & $z$ & $\begin{array}{c}\mathrm{ACS} \\
\mathrm{F} 435 \mathrm{~W}\end{array}$ & $\begin{array}{c}\text { ACS } \\
\text { F606W }\end{array}$ & $\begin{array}{c}\text { ACS } \\
\text { F775W }\end{array}$ & $\begin{array}{c}\text { ACS } \\
\text { F850LP }\end{array}$ & $\begin{array}{l}\text { WFC3 } \\
\text { F105W }\end{array}$ & $\begin{array}{l}\text { WFC3 } \\
\text { F125W }\end{array}$ & $\begin{array}{l}\text { WFC3 } \\
\text { F160W }\end{array}$ & IRAC1 & IRAC2 \\
\hline 631 & 4.0 & $29.59 \pm 0.35$ & $26.87 \pm 0.02$ & $26.78 \pm 0.38$ & $26.62 \pm 0.18$ & $26.49 \pm 0.07^{\mathrm{a}}$ & $\ldots$ & $26.76 \pm 0.10^{\mathrm{a}}$ & $27.17 \pm 0.37$ & $27.16 \pm 0.44$ \\
\hline 712 & 5.2 & $31.48 \pm 2.01$ & $29.66 \pm 0.25$ & $28.06 \pm 0.84$ & $27.12 \pm 0.04$ & $\ldots$ & $\ldots$ & $\ldots$ & $28.05 \pm 0.85$ & $>27.45$ \\
\hline 4442 & 5.8 & $>32.96$ & $>31.64$ & $>31.40$ & $>29.82$ & $29.35 \pm 0.06$ & $29.16 \pm 0.05$ & $29.45 \pm 0.05$ & $>28.53$ & $>28.07$ \\
\hline 5183 & 4.8 & $>31.76$ & $>31.40$ & $28.61 \pm 1.25$ & $27.97 \pm 0.09$ & $28.18 \pm 0.05$ & $28.15 \pm 0.05$ & $28.34 \pm 0.05$ & $>28.13$ & $>27.85$ \\
\hline 5225 & 5.4 & $>28.88$ & $>28.29$ & $>26.21$ & $25.93 \pm 0.04$ & $25.94 \pm 0.05$ & $25.87 \pm 0.05$ & $25.95 \pm 0.05$ & $26.51 \pm 0.28$ & $25.86 \pm 0.26$ \\
\hline 6139 & 4.9 & $>32.92$ & $>26.97$ & $25.96 \pm 0.45$ & $25.66 \pm 0.02$ & $25.56 \pm 0.05$ & $25.57 \pm 0.05$ & $25.64 \pm 0.05$ & $26.35 \pm 0.23$ & $27.10 \pm 0.39$ \\
\hline 9040 & 4.9 & $>30.14$ & $>28.33$ & $>26.17$ & $26.23 \pm 0.05$ & $26.15 \pm 0.05$ & $26.28 \pm 0.05$ & $26.31 \pm 0.05$ & $>28.45$ & $>28.36$ \\
\hline 9340 & 4.7 & $>33.04$ & $>31.40$ & $28.25 \pm 0.77$ & $27.69 \pm 0.07$ & $27.86 \pm 0.05$ & $28.61 \pm 0.05$ & $28.02 \pm 0.05$ & $>27.48$ & $>27.16$ \\
\hline 9487 & 4.1 & $>30.07$ & $>28.24$ & $27.16 \pm 0.03$ & $27.27 \pm 0.05$ & $27.13 \pm 0.05$ & $27.41 \pm 0.05$ & $27.42 \pm 0.05$ & $27.47 \pm 0.47$ & $>27.93$ \\
\hline $\mathrm{L} 7-01^{\mathrm{b}}$ & 7 & $>29.89$ & $>31.20$ & $>29.31$ & $28.57 \pm 0.7$ & $27.37 \pm 0.29$ & $27.18 \pm 0.18$ & $26.93 \pm 0.18$ & $25.52 \pm 0.25$ & $26.08 \pm 0.72$ \\
\hline L8-01 ${ }^{\mathrm{b}}$ & 8 & $>29$. & $>30.50$ & $>29$. & $>29$ & $>30.89$ & $27.38 \pm 0.21$ & $27.47 \pm 0.20$ & $>27.71$ & $>26.92$ \\
\hline A383-iD $1^{\mathrm{c}}$ & 6. & $\begin{array}{l}\cdots \\
\ldots\end{array}$ & $\begin{array}{l}\ldots \\
\ldots\end{array}$ & $>26.48$ & $\begin{array}{l}24.54 \pm 0.12 \\
25.63 \pm 0.14^{\mathrm{e}}\end{array}$ & $24.65 \pm 0.05^{\mathrm{d}}$ & $24.57 \pm 0.08$ & $24.66 \pm 0.05$ & $23.06 \pm 0.08$ & $22.83 \pm 0.08$ \\
\hline$A 1689-z D 1^{f}$ & 7.6 & $\ldots$ & $>27.80$ & $>27.80$ & $>27.50$ & $\ldots$ & $25.30 \pm 0.10^{g}$ & $24.70 \pm 0.10^{\mathrm{g}}$ & $24.20 \pm 0.30$ & $23.90 \pm 0.30$ \\
\hline A1703-iD $1^{h}$ & 6. & $\ldots$ & $>28.20$ & $26.80 \pm 0.30$ & $24.20 \pm 0.10$ & $\ldots$ & $24.00 \pm 0.10$ & $23.90 \pm 0.10$ & $23.10 \pm 0.30$ & $23.50 \pm 0.40$ \\
\hline A2218-iD1 ${ }^{\mathrm{h}}$ & 6.7 & $\ldots$ & $>27.60$ & $>27.20$ & $25.10 \pm 0.10$ & $\ldots$ & $24.30 \pm 0.10$ & $24.10 \pm 0.10$ & $23.70 \pm 0.30$ & $23.90 \pm 0.30$ \\
\hline CL0024-iD1 ${ }^{\mathrm{h}}$ & 6.5 & $\cdots$ & $>28.10$ & $>27.90$ & $26.00 \pm 0.20$ & $\cdots$ & $25.10 \pm 0.10$ & $25.00 \pm 0.10$ & $24.40 \pm 0.20$ & $24.40 \pm 0.30$ \\
\hline CL0024-zD1 ${ }^{\mathrm{h}}$ & 6.6 & $\ldots$ & $>28.10$ & $>27.90$ & $27.30 \pm 0.80$ & $\ldots$ & $26.00 \pm 0.20$ & $25.60 \pm 0.20$ & $24.50 \pm 0.50$ & $24.80 \pm 0.50$ \\
\hline
\end{tabular}

Notes. Upper limits are $1 \sigma$.

${ }^{\text {a }}$ HST/NICMOS measurements with F110W Filter.

b Sample object with photometric redshift from Labbé et al. (2010).

${ }^{c}$ Lensed object with possible spectroscopic confirmation from Richard et al. (2011).

d WFC3 measurements with F110W Filter.

e ACS/WFC measurements with F814W filter.

${ }^{f}$ Lensed object with photometric redshift from Bradley et al. (2008).

g HST/NICMOS measurements with F110W and F160W Filter.

${ }^{\mathrm{h}}$ Lensed object with photometric redshift from Zheng et al. (2009).

both $95 \%$ and $68 \%$ credible regions in this paper so that the reader can appreciate how the two differ and how one might be misled when $68 \%$ intervals are examined.

\subsection{Applied Technique}

Our implementation of an MCMC SED analysis, $\pi \mathrm{MC}^{2}$, is written in Python and based on the freely available pyMC module (Patil et al. 2010). The use of the $\pi \mathrm{MC}^{2}$ Python package allowed us to implement the more advanced MCMC variants and allows $\pi \mathrm{MC}^{2}$ to run on various platforms such as Linux and OSX with no modifications. $\pi \mathrm{MC}^{2}$ generates models using either Bruzual \& Charlot (2003), S. Charlot \& G. Bruzual (2012, in preparation), or Maraston (2005) stellar population libraries, using one (SSP), two (SSP2) stellar instantaneous populations, or an exponentially decaying star formation history (SFH) model (EXP). We also included the effect of continuum and nebular lines emissions, following the recipe described in Nilsson et al. (2007). The nebular continuum emission was taken from Starburst99 (Leitherer et al. 1999). The emission line list was taken from Inoue (2011) and includes 119 emission lines up to the rest-frame wavelength of $10,000 \AA$. The nebular and line emission were scaled with the $\mathrm{H} \alpha$ flux and the number of ionizing photons predicted by the Bruzual \& Charlot (2003) models. The nebular emission models were interpolated to arbitrary values of metallicities and ages. The input templates that are used with $\pi \mathrm{MC}^{2}$ are defined only at discrete ages and metallicities and these templates are interpolated to produce models with arbitrary stellar age and metallicities. We usually used flat priors for all parameters which assign equal likelihood to all allowed parameter values. This is a conservative choice of priors that are appropriate for most cases where we have no a priori information about the nature of the object we are analyzing. We have also used non-flat priors, such as semiGaussian for the extinction, to check that our results were not being biased by the choice of a flat prior or by the bounding values we chose for these parameters. We used either linear, natural logarithmic, or base ten logarithmic distributions for the stellar ages and found that these did not affect the final posterior distributions. The only additional assumptions that were made are that the stellar ages are constrained to be smaller then the age of the universe at a given redshift $z$, and that the young stellar component in the SSP2 models is constrained to be younger than the older stellar population.

The model parameters that can be varied are the population ages; the relative ratio between the old and young stellar populations (parameterized as the fraction of the total stellar mass that is in the form of the older stellar population); the metallicities of the young or old stellar populations; the extinction $\left(A_{\mathrm{V}}\right.$, based on Calzetti et al. 2000 law); the halflife $\tau$ value in the case of exponential (EXP) models; and the nebular emission escape fraction, $f_{\text {esc }}$. A value of one for the escape fraction results in no nebular emission while a value of zero results in the maximum amount of nebular continuum and line emission in the simulated spectra. The stellar mass is computed deterministically by scaling each computed model to the observations, similar to what was done in Nilsson et al. (2011) and Acquaviva et al. (2011). When computing the mass scale and likelihood between model and observations, we take into account photometric bands in which flux is not detected and the upper limit to the flux in these bands. The upper limit to the flux in these bands is used as part of a penalized likelihood similar to what was done in Lai et al. (2007) and Pirzkal et al. (2007). We have also allowed for the stellar mass 
to be varied as a stochastic parameter but saw no effect in doing so, expect that the chains could sometimes take longer to converge.

The input to $\pi \mathrm{MC}^{2}$ is a text file that contains (1) the measured $\mathrm{AB}$ magnitudes or fluxes (magnitudes are converted to fluxes internally by $\pi \mathrm{MC}^{2}$ ); (2) errors (real, or upper limits); and (3) the list of all parameters with ranges to probe. About 10 iterations per second can be generated using $\pi \mathrm{MC}^{2}$ with today's typical desktop computer. A 10,000 iteration chain can be generated in about 30 minutes, assuming a step rejection of about $50 \%$, or about 2 hr per object when running multiple chains of a few tens of thousand iterations each. The stepping method found to work best is the Metropolis step, as described in the pyMC documentation. We have also had equally good results using an Adaptive Metropolis step once the chain had converged. The MCMC approach is highly parallelized and we used $\pi \mathrm{MC}^{2}$ on both a Linux cluster of 88 processor and a 36 node OSX Xgrid cluster.

\subsection{MCMC and Convergence}

The Bayesian foundation of MCMC guarantees that credible regions can be derived if a chain has converged and if the MCMC chain(s) contains a sufficient number of iterations. There is however nothing inherent in the method that ensures that the chain actually converges toward the best fit to the observations. It is the responsibility of the user to check the quality of the fit and convergence of each MCMC chain. We used several methods to do this, some empirical and some based on statistical methods. To empirically check for convergence, we started by simply inspecting the traces and histograms of the individual parameters contained in each MCMC chain. Examples of such plots are available in Patil et al. (2010). One big advantage of the MCMC approach is that the method allows for several MCMC chains to be generated independently and in parallel and be combined later, after they are checked for convergence. We could then assemble a final MCMC chain by discarding chains that did not converge or resulted in poor fits (with relatively low maximum likelihood) and combining the rest of the chains together. We found it necessary, especially in cases where the input models were complex, to produce several dozen independent short (few thousand iterations each) MCMC chains. The chains converging to the highest likelihood were then restarted and extended to several tens of thousands of iterations each. In some cases, we simply generated new independent chains with initial values set to the currently best-fitting model so that convergence time was minimized. We found that examining the intra chain standard deviation (for a given parameter in the chain) and comparing it to the inter chain dispersion (using several independent chains) allowed us to determine where each chain had converged. More formally, we also routinely checked for convergence of a particular chain parameter by computing the mean and variance of segments of a chain, from the beginning to the end, following the work of Geweke (1992). We also made use of another diagnostic (Raftery \& Lewis 1995) which allowed us to derive and estimate the number of iterations to discard at the beginning of the chain, and the minimum number of iterations required to estimate the credible region of a parameter to a given accuracy. More information about these procedures is described in Patil et al. (2010). Throughout this paper, we conservatively produced MCMC chains that were significantly longer than the number derived from the Raftery-Lewis method to estimate a $95 \%$ credible interval with a $5 \%$ precision.
While painstaking, we must stress that this convergence checking is both crucial and necessary if one aims to derive useful credible intervals. Failure to do this leads to biased credible intervals. We further address the issue of whether we can actually trust the implied meaning of the $95 \%$ credible regions in Section 3.3.1.

\section{CALIBRATION OF $\pi \mathrm{MC}^{2}$}

Before applying $\pi \mathrm{MC}^{2}$ to real data, it is useful to examine how well the method works by applying $\pi \mathrm{MC}^{2}$ to a series of simple simulated observations. The tests presented here do not constitute an exhaustive set, but we selected them among the many tests we performed because they illustrate how well $\pi \mathrm{MC}^{2}$ performs. In Section 3.1, we start by first examining the effects of the size of the photometric error bars, and for now ignoring the real effects of photometric noise that displaces the observations away from the fiducial values. We then examine the effect of increasing the number of fitted parameters, using more complex models in Section 3.2. Finally, we add real photometric noise to determine the effect of displacing the observed fluxes away from their fiducial values, in Section 3.3. When generating simulated observations, we used BC03 models as input and produced simulated fluxes in the following bandpasses: Advanced Camera for Surveys (ACS) F435W, F606W, F775W, F814W, F850LP filters, the WFC3 F105W, F110W, F125W, F160W filters, and the IRAC 3.6 and $4.5 \mu \mathrm{m}$ channels. This choice was driven by the filters used in Section 4 where we apply $\pi \mathrm{MC}^{2}$ to real observations.

\subsection{The Simplest Case: No Real Noise, Increasing the Size of the Error Bars Only}

We begin with a very simple case: noiseless observations of a $10^{10} M_{\odot}$ galaxy at a redshift of 4.5 . This galaxy has a single 0.6 Gyr old stellar population, a global extinction of $A_{\mathrm{V}}=0.2$, and a stellar metallicity of $Z=0.001$. We tested $\pi \mathrm{MC}^{2}$ with many test objects with ages ranging from very young to old, with little to high extinction, and with low to high metallicities. In this paper, we chose this example because these types of objects are not easily modeled using conventional SED fitting routines. The aim of this section is to demonstrate how a limited set of photometric bands, combined with larger error bars, results in a significant broadening of the credible regions of the physical characteristic of an object. This is an expected result as it is intuitively obvious that as error bars are widened, a larger number of models become consistent with the observations. Furthermore, the inter dependency and degeneracy between model parameters also lead to a widening of the credible regions. We illustrate this by showing the results obtained from using error bars that are $1 \%$ of the flux, $5 \%$ of the flux, and finally error bars that are the same size as those in the Hubble Ultra-Deep Field (HUDF; Beckwith et al. 2006).

Figure 1 shows several diagnostic plots. First, in panel (a), we plot the models that are contained in the MCMC chain. As we outlined earlier, an MCMC chain will spend more time in higher likelihood regions. Likelier models will appear more often in the MCMC chain. This plot clearly shows that most of the models clearly fit within the photometric error bars. As already mentioned in Section 2.3, MCMC provides a formalism to estimate the errors associated with a posterior estimate of each given model parameter, but does not guarantee that a true likelihood maximum will be found within a preset number of iterations. Indeed, chains that are too short and 

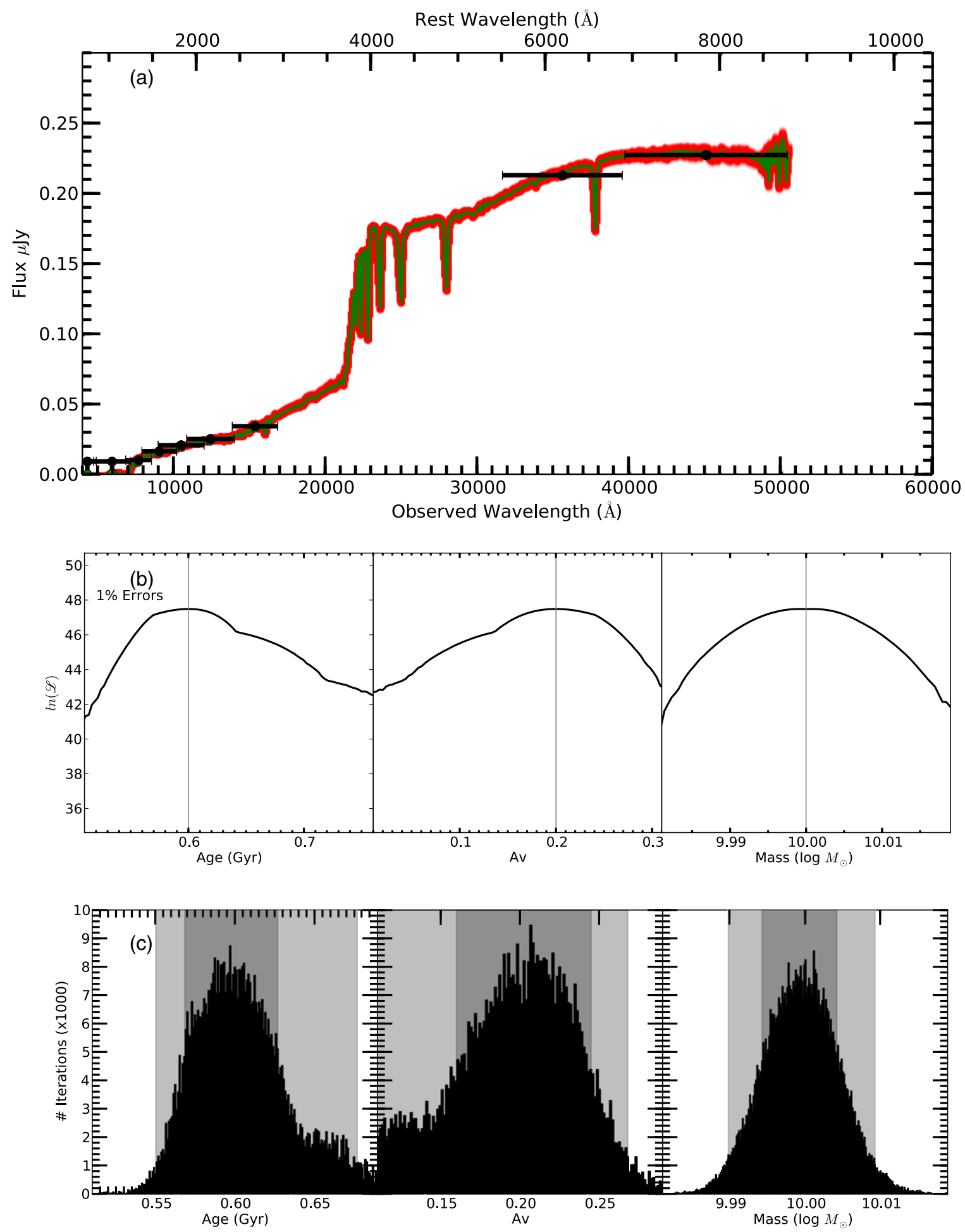

Figure 1. Observed simulated single $0.6 \mathrm{Gyr}$ old stellar population object at $z=4.5$ with a mass of $10^{10} M_{\odot}$ and $A_{\mathrm{V}}=0.2$, shown with exact photometric values but using $1 \%$ error bars (black error bars) in panel (a). The best-fitting model, toward which the chains converged, is shown using a thick solid green line. Models from the MCMC chain are shown in red and the redder areas correspond to the region of the SED where more models happen to lie. Panel (b) shows plots of the log likelihood as a function of individual model parameters. These three plots show how the maximum likelihood (shown with a gray vertical bar) is relatively well defined. Panel (c) shows the posteriori probability distributions of the model parameters, as determined from the $\pi \mathrm{MC}^{2}$ results. We also show the corresponding $66 \%$ and $95 \%$ credible intervals, shown in light and dark gray, respectively.

have not converged to the true maximum likelihood will lead to erroneous parameter estimates. In panel (b), we show the maximum value of the likelihood as a function of model parameter (marginalizing all others). This likelihood function is well behaved, smooth, and clearly peaked. Finally, panel (c) shows the probability distribution function for each model parameter. From these, we derive our $95 \%$ credible region and can state (with a 95\% confidence) that this object is a $\log$ (mass) of $10 ._{-0.01}^{0.01}$ galaxy with a $0.61_{-0.06}^{0.07}$ Gyr stellar population and an extinction of $0.19_{-0.10}^{0.08}$. While this is in excellent agreement 

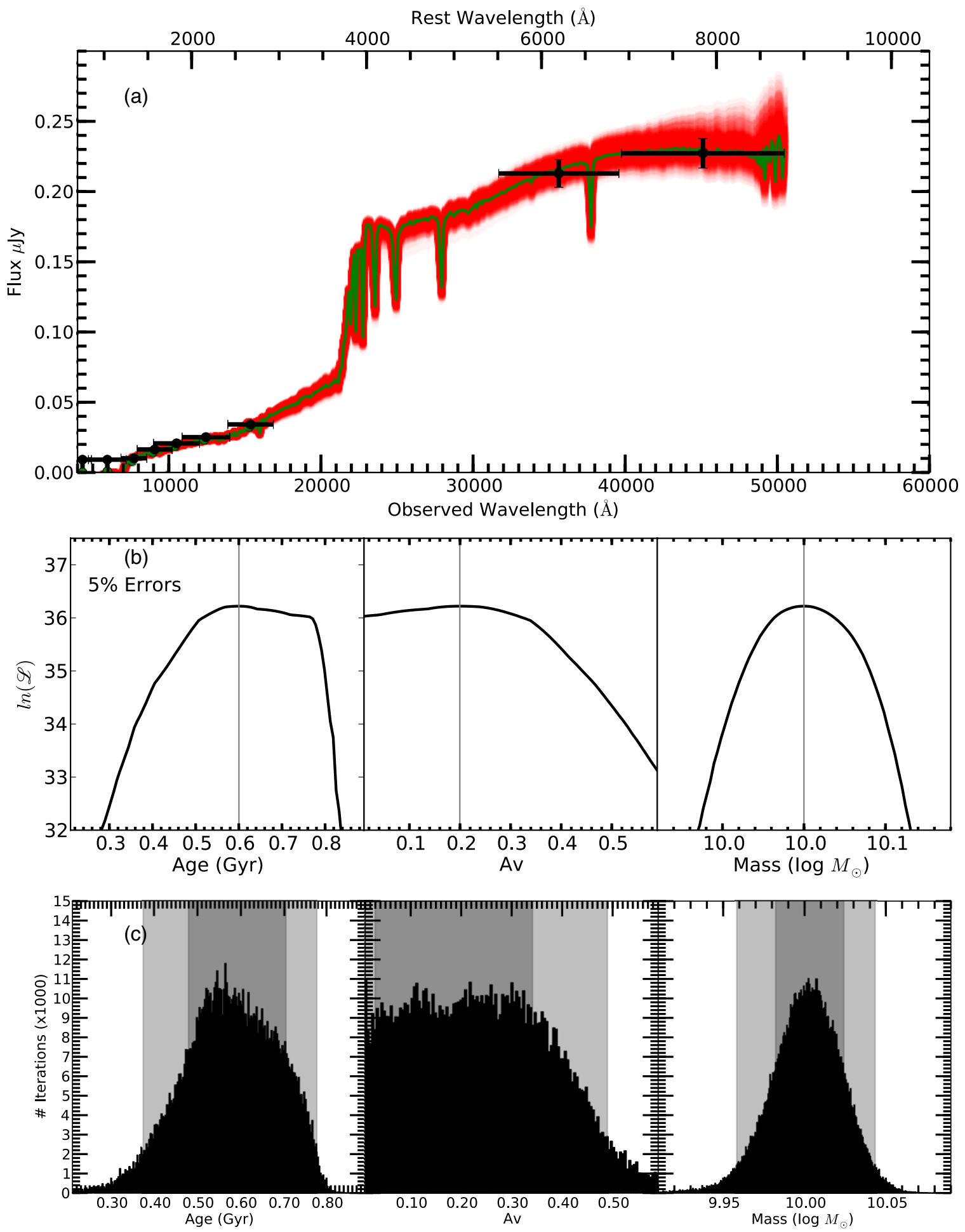

Figure 2. Same Figure 1 but showing the effect of allowing for larger error bars in the input photometry. We now associate a uniform 5\% uncertainty with each photometric band. The immediate effect of just allowing for larger uncertainties, a broadening of the PDF of each of the model parameters, is clearly shown here. Larger error bars allow for a larger number of combination of stellar ages, extinction, and stellar mass to statistically match the observations.

with the fiducial values listed at the beginning of this section, it appears that some model parameters are better constrained than others (e.g., age and mass versus $A_{\mathrm{V}}$ ).

In Figure 2, we show the effect of increasing the size of the error bars to 5\%. Everything else remains the same. The figure demonstrates that increasing the size of the error bars flattens the shape of the maximum likelihood functions shown in panel (b), particularly for extinction. Panel (c) shows much broader PDF for each parameter. It can only be stated with confidence that this object has $\log$ (mass) $=10.0 .04$, an age of $0.58_{-0.21}^{0.19} \mathrm{Gyr}$, and an extinction of $0.23_{-0.23}^{0.25}$. This is a significant broadening of the credible regions. It is particularly important to note that the PDF for $A_{\mathrm{V}}$ and age are now very non-Gaussian.

The two previous tests were performed with error bars that are significantly smaller than those seen in deep surveys (e.g., HUDF and GRAPES). In Figure 3, we now consider a more 

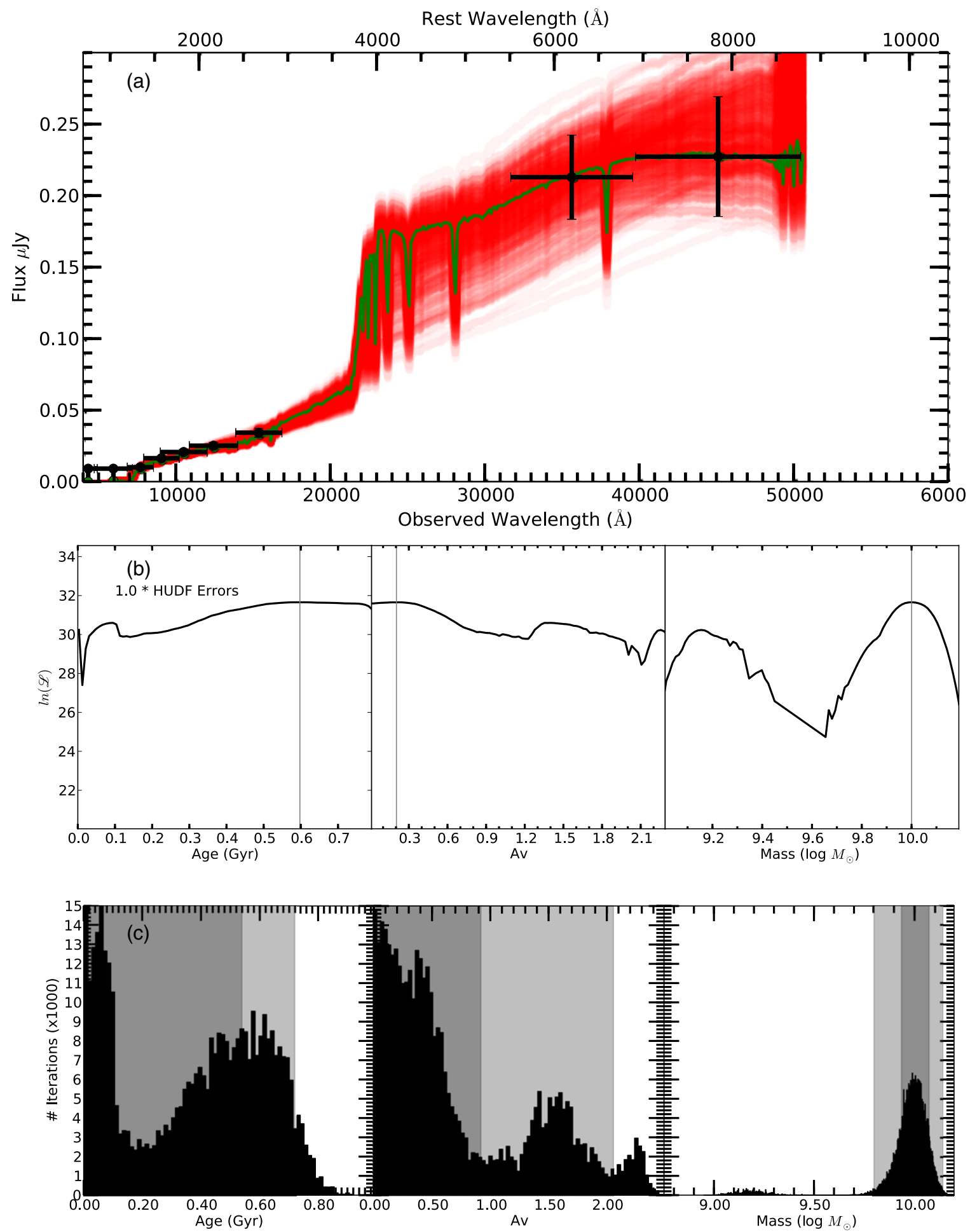

Figure 3. Same as Figures 1 and 2 but we now adopt more realistic larger error bars in the input photometry. While we assume a uniform $1 \%$ error in each band in Figure 1 and 5\% in Figure 2, we now adopt uncertainties of 5\% in the ACS bands, 10\% in the WFC3 bands, and 15\% and 20\% in the two IRAC bands. The effect of just assuming larger uncertainties is again very clearly shown here. We are now in a regime where we can see that the PDFs of the stellar ages, extinction, and, to a much smaller extent, stellar mass now take on distinctively non-Gaussian shapes (panel (c)). Determining the stellar ages and extinction of this object has now become more difficult as the PDFs for these quantities are now very wide.

realistic case where the size of the error bars is set to $5 \%$ for the ACS bands, $10 \%$ for the WFC 3 bands, and $15 \%$ for IRAC1 and $20 \%$ for the IRAC2. These values approximate the accuracy level of the data available for the HUDF. As panels (b) and (c) show, these realistic error levels lead to much more complex maximum likelihood functions with multiple peaks and PDFs that are very non-Gaussian. We now estimate that the galaxy has an age of $0.42_{-0.41}^{0.30} \mathrm{Gyr}$, an extinction $A_{\mathrm{V}}$ of $0.48_{-0.48}^{1.6}$, and a mass of $10 ._{-0.19}^{0.15}$. The larger errors allow for two distinct models: young and dusty $\left(<0.2 \mathrm{Gyr}\right.$ old, $\left.A_{\mathrm{V}}>1.0\right)$ and the fiducial one ( $>0.2 \mathrm{Gyr}$ old, $A_{\mathrm{V}}<0.6$ ). The combination of model degeneracy and large error bars strongly limits one's 

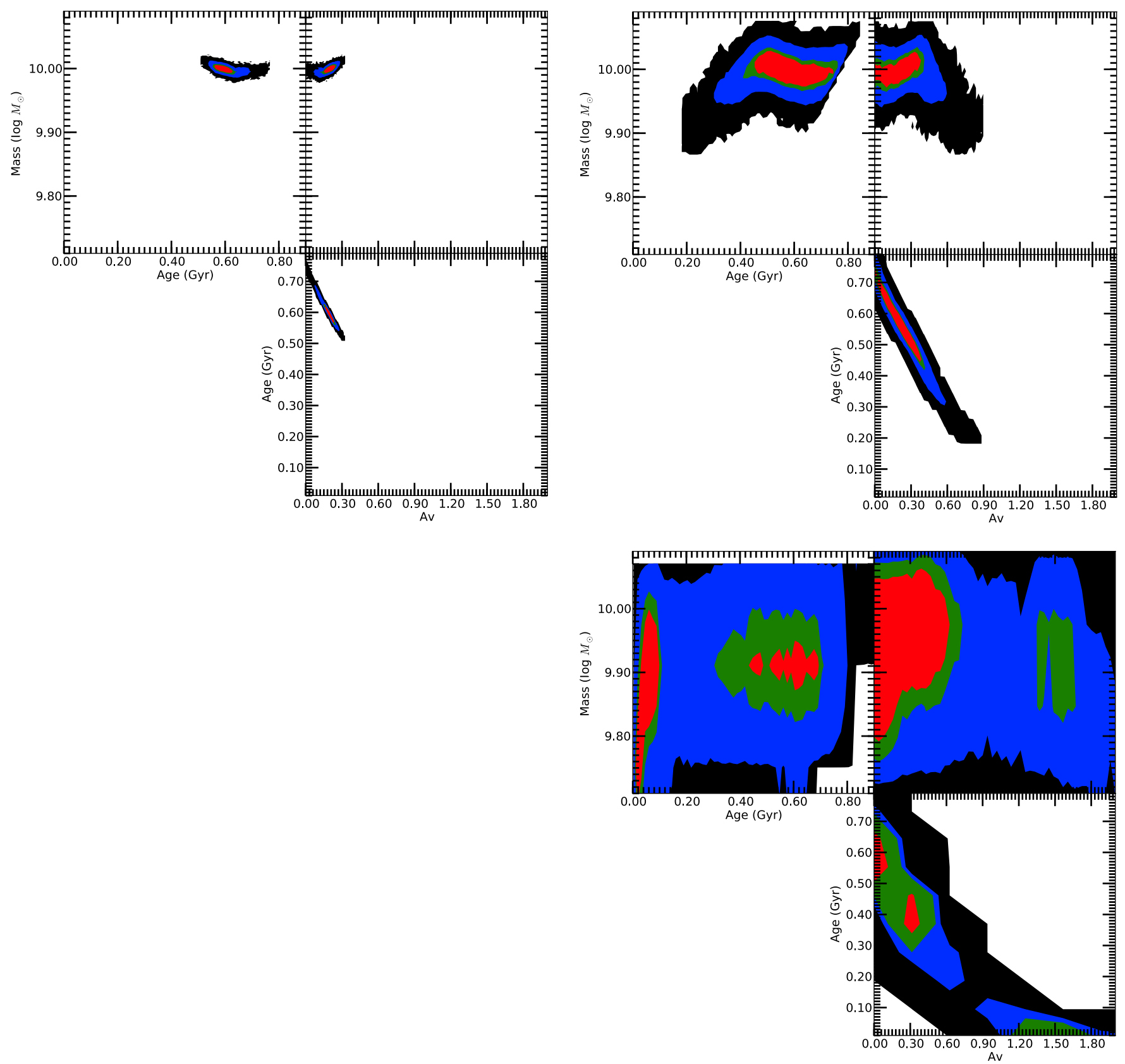

Figure 4. Two-dimensional model parameter probability distribution obtained with our MCMC SED fitting method and the simulated data shown in Figure 1 (1\% uncertainties, top left panels), Figure 2 (5\% uncertainties, top right panels), and Figure 3 (typical HUDF error levels in each photometric band, bottom right panels). The red, green, blue, and black regions are the 50\%, 68\%, 95\%, and $99 \%$ credible regions, respectively. All three cases show credible regions that included the fiducial stellar age of $0.6 \mathrm{Gyr}$, extinction of $A_{\mathrm{V}}=0.2$, and stellar mass of $10^{10} M_{\odot}$. However, the size of the credible regions increased and degeneracy between stellar ages and extinction also increases as the level of photometric uncertainty is increased from $1 \%$ to $5 \%$ and then finally to more realistic HUDF levels.

ability to derive physical parameters using SED fitting. The increase in model degeneracy as the size of the error bars become larger is shown in Figure 4. In this figure, we plot two-dimensional probability densities where the degeneracy between stellar age and extinction are clear. As one goes from using $1 \%$ error bars (top left panel) to 5\% (top right panel) to HUDF level error bars (bottom right panel), the 95\% credible interval (shown in blue) first stretches out and finally splits into multiple subregions of acceptable parameters. Note that even the interval of acceptable stellar mass increases as different stellar populations with different mass-to-light ratios become acceptable.

\subsection{More Complex Cases: Increasing the Number of Free Parameters}

We have shown in Section 3.1 that model degeneracy limits our ability to constrain the physical characteristics of even the most basic galaxies. We now test $\pi \mathrm{MC}^{2}$ with increasingly complex models. As noted in Section 1, one advantage of MCMC is that it provides a computationally efficient way to compare observations to arbitrarily complex models. But, as we consider more complex models, the degeneracy between the various model parameters can only lead to wider posterior credible intervals. We first begin with metallicity in Section 3.2.1, then 


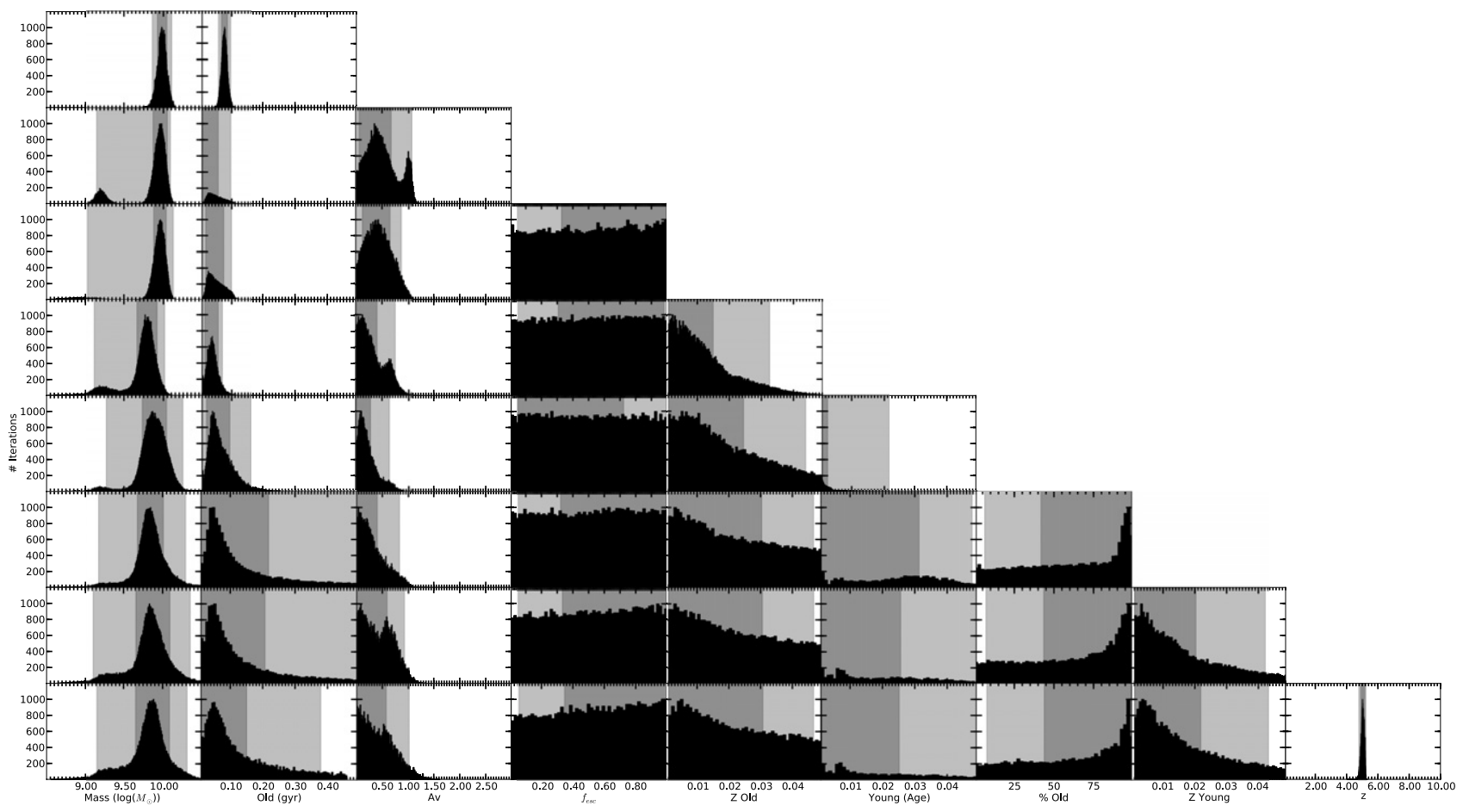

Figure 5. Model parameters resulting from applying $\pi \mathrm{MC}^{2}$ using an increasingly complex model. We start (top row) by only allowing the mass and stellar population age to be varied. Each subsequent row then shows the result when adding another free parameter and running $\pi \mathrm{MC}^{2}$. As input, we have used a simulated galaxy at $z=5$ with $99 \%$ of stars 0.1 Gyr old with metallicity $Z=0.001$ and with $1 \%$ of stars 50 Myr old with metallicity $Z=0.02$ (solar). The extinction is $A_{\mathrm{V}}=0.2$ and the total stellar mass is $10^{10} M_{\odot}$.

add a second stellar population and nebular emission to create more complex models in Section 3.2.2.

\subsubsection{Adding Metallicity}

We now add metallicity to the earlier parameters of mass, age, and extinction, and test how $\pi \mathrm{MC}^{2}$ handles this using the simulated observations described in Section 3.1. Using a $1 \%$ photometric uncertainty across all bands, we derive the $95 \%$ credible regions of $0.61_{-0.05}^{0.06} \mathrm{Gyr}, A_{\mathrm{V}}=0.19_{-0.07}^{0.09}, Z=$ $0.001_{-0.0001}^{0.001}$, and $\log$ (mass) of $10 ._{-0.02}^{0.01}$. This is very similar to the earlier results in which metallicity remained fixed. The only differences are a slightly wider stellar mass uncertainty and a slight trend toward lower stellar masses. Increasing the uncertainties to the $5 \%$ levels yields $0.52_{-0.38}^{0.20} \mathrm{Gyr}$, $A_{\mathrm{V}}=0.21_{-0.20}^{0.51}, Z=0.002_{-0.0001}^{0.01}$, and $\log$ (mass) of $10 ._{-0.11}^{0.06}$. These credible regions are now approximately twice as wide as the ones derived in Section 3.1 using 5\% error bars. Finally, using HUDF level errors we obtain $0.14_{-0.11}^{0.34} \mathrm{Gyr}, A_{\mathrm{V}}=$ $0.79_{-0.79}^{0.60}, Z=0.01_{-0.01}^{0.02}$, and $\log$ (mass) of $9.9_{-0.16}^{0.16}$. These credible regions are once again several times wider than those derived earlier using HUDF error bar sizes but holding metallicity fixed. Model degeneracy has a strong impact on the size of the posterior credible regions for all parameters and wrongly assuming an a priori metallicity for a galaxy may lead to credible intervals that are artificially small.

\subsubsection{Increasingly Complex Models}

We conclude this section by showing the effect upon credible intervals for eight increasingly complex input models. The simulated galaxy is a $10^{10} M_{\odot}$ galaxy with two stellar populations of age $0.1 \mathrm{Gyr}(Z=0.0001)$ and $50 \mathrm{Myr}(Z=0.02) .99 \%$ of the stellar population is old with $A_{\mathrm{V}}=0.2$. These values and population ratios were chosen so that the light produced by the younger stellar population would not be the dominant contribution to the stellar light of the simulated galaxy. Unlike the previous section, we now only consider realistic HUDF-sized error bars. Figure 5 shows the results of applying $\pi \mathrm{MC}^{2}$ using eight different parameters for the same set of simulated observations. Each row of Figure 5 shows the credible regions produced by $\pi \mathrm{MC}^{2}$ for a given model. We start with a simple stellar population fit (SSP), allowing only the stellar age and mass to vary (top row). We then allow for extinction to be varied (row 2). In turn, we then add the escape fraction for the nebular emission; metallicity; a second, older stellar population (SSP2); and finally set redshift as a free parameter. This figure illustrates how continually adding model parameters produces increasingly non-Gaussian PDFs. This, in turn, weakens the constraints on the models. However, some parameters, such as mass, extinction, old stellar population age, and redshift can still be reasonably constrained. While others, such as nebular emission and metallicity cannot be constrained. Instead, they become "nuisance" parameters, which add little to the final results. For example, the nebular emission cannot be constrained. Nominally, nebular emission contributes relatively little to objects with ages $>10 \mathrm{Myr}$ (Reines et al. 2010). Although $\pi \mathrm{MC}^{2}$ is unable to constrain this parameter, it does not seriously affect the quality of the posterior credible intervals of other parameters.

\subsection{Impact of Real Photometric Errors}

We now address the issue of real photometric noise and how it may affect the MCMC-derived posterior credible regions. The tests performed in Section 3.1 used noiseless data. The error bars were simply some percentage of the flux in each filter, while the flux value itself never varied. In the case of testing real photometric errors, the simulated fluxes in each filter are taken to be within some range of their "true" value in each iteration of the model. For example, if 10 iterations of a model 


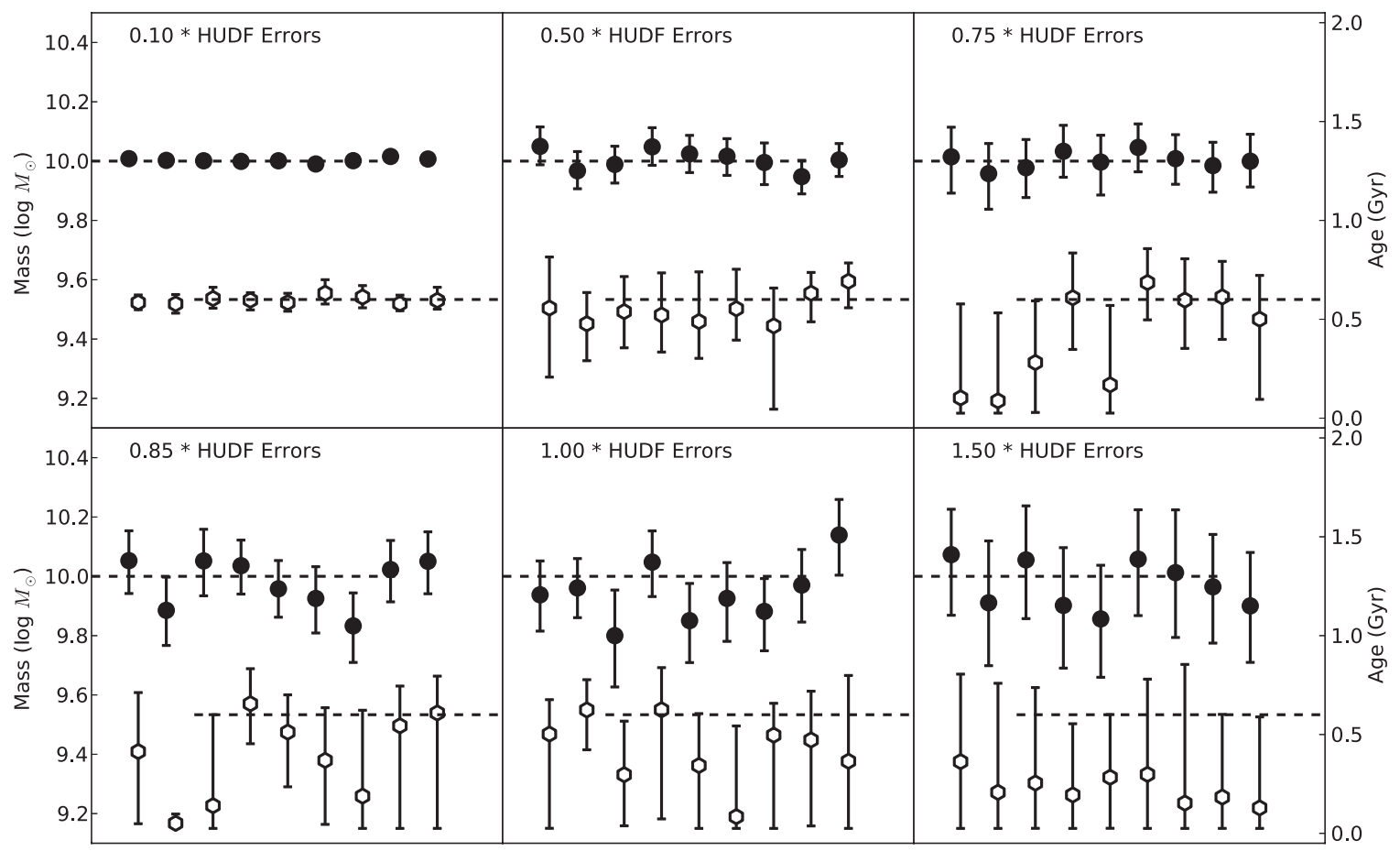

Figure 6. Credible 95\% interval for the stellar population mass (dark circles) and stellar population age (white circles) as a function of photometric error. Each panel shows 10 independent simulations and the resulting 95\% credible region for the $\log$ (mass) and stellar age of the object. Different panels show the results obtained under different photometric accuracy, ranging from 0.1 to 1.5 times that of typical high-redshift HUDF error levels. The input model is a $10^{10} M_{\odot}$ galaxy with a single stellar population $0.6 \mathrm{Gyr}$ old and $A_{\mathrm{V}}=0.2$, and is shown using dashed lines.

are run, each of the 10 iterations will have a different value, as determined by a random Gaussian variate, for the flux. Each iteration will be within some percent of the "true" value. This is a more realistic approach, as repeated observations of the same object will always vary to some degree, and the observer never knows the "true" value. In order for $\pi \mathrm{MC}^{2}$ to be useful, we must check its behavior and the accuracy of the credible intervals it derives using more realistic test cases.

\subsubsection{Testing the Reliability of the Posterior Credible Regions}

For the posterior credible regions to be useful, they must translate to a simple reality: a $95 \%$ credible region should include the fiducial value nearly $95 \%$ of the time. We tested this by generating 200 simulated observations of a $10^{10} M_{\odot}$ galaxy with a $0.6 \mathrm{Gyr}$ old stellar population and $A_{\mathrm{V}}=0.2$. We included the effect of photometric noise (assuming our previously defined HUDF error levels) and produced a set of 200 slightly different input SEDs to be analyzed using $\pi \mathrm{MC}^{2}$. Examining the $95 \%$ posterior credible regions and comparing them to the fiducial model parameters we find that input mass of $10^{10} M_{\odot}$ was in the range predicted by the posterior $95 \%$ credible interval for stellar mass 183 out of 200 times (92\%). Similarly, the fiducial stellar age and extinction were contained in the corresponding posterior credible regions $90 \%$ and $94 \%$ of the time, respectively. This clearly demonstrates that we can rely on $\pi \mathrm{MC}^{2}$ posterior credible regions to constrain physical characteristics of galaxies.

\subsubsection{Photometric Noise Level and Its Effect on Posterior Credible Regions}

We have shown in Section 3.1 that the size of error bars has an effect on the width and shape of the posterior credible regions. We now examine for two distinct cases how decreasing the signal to noise $(\mathrm{S} / \mathrm{N})$ of observations can change the derived posterior credible regions. We examine two test cases at redshift 4.75. This redshift is typical for the sources in Pirzkal et al. (2007). Both cases have a stellar mass of $10^{10} M_{\odot}$ and an extinction of $A_{\mathrm{V}}=$ 0.2 . The first case is a galaxy with a metallicity of $Z=0.001$ and a stellar population that is $0.60 \mathrm{Gyr}$. The second case is a galaxy with a 50 Myr stellar population. We generated 10 iterations for each of these simulated galaxies with six increasing levels of random noise added (0.1.HUDF, 0.5 HUDF, 0.75.HUDF, $0.85 \cdot \mathrm{HUDF}, 1.0 \cdot \mathrm{HUDF}$, and $1.5 \mathrm{HUDF})$. The $95 \%$ posterior credible regions we derived are shown in Figures 6 and 7.

At photometric error levels of $0.1 \mathrm{HUDF}$, we can confidently estimate the stellar masses in both models (top left panels in Figures 6 and 7). However, by the time photometric errors match those of the HUDF, the posterior credible mass intervals span a factor of 3.0. As more errors are added to each photometric band, the possibility of deriving a biased credible interval also increases. Both figures demonstrate that the posterior credible intervals do include the fiducial parameter values. However, one can also see in Figure 6 that increasing the sizes of the photometric errors leads to a larger number of acceptable models with younger stellar populations. This is because an observed SED can be made redder from either the presence of an old stellar population or by increasing $A_{\mathrm{V}}$. On the other hand, a very young, blue population with little or no extinction has a very distinct SED shape. It is difficult to match anything other than a young SED to this type of population.

\subsubsection{The Impact of Adding the $3.6 \mu \mathrm{m}$ and $4.5 \mu \mathrm{m}$ IRAC Channels}

At the redshifts considered here, IRAC observations using Channel $1(3.6 \mu \mathrm{m})$ and Channel $2(4.5 \mu \mathrm{m})$ are often the only means we have to probe the rest-frame optical colors of highredshift galaxies. It is at these wavelengths that we may hope to constrain the contribution of any old stellar population(s). In addition, these wavelengths probe the red side of the Balmer 


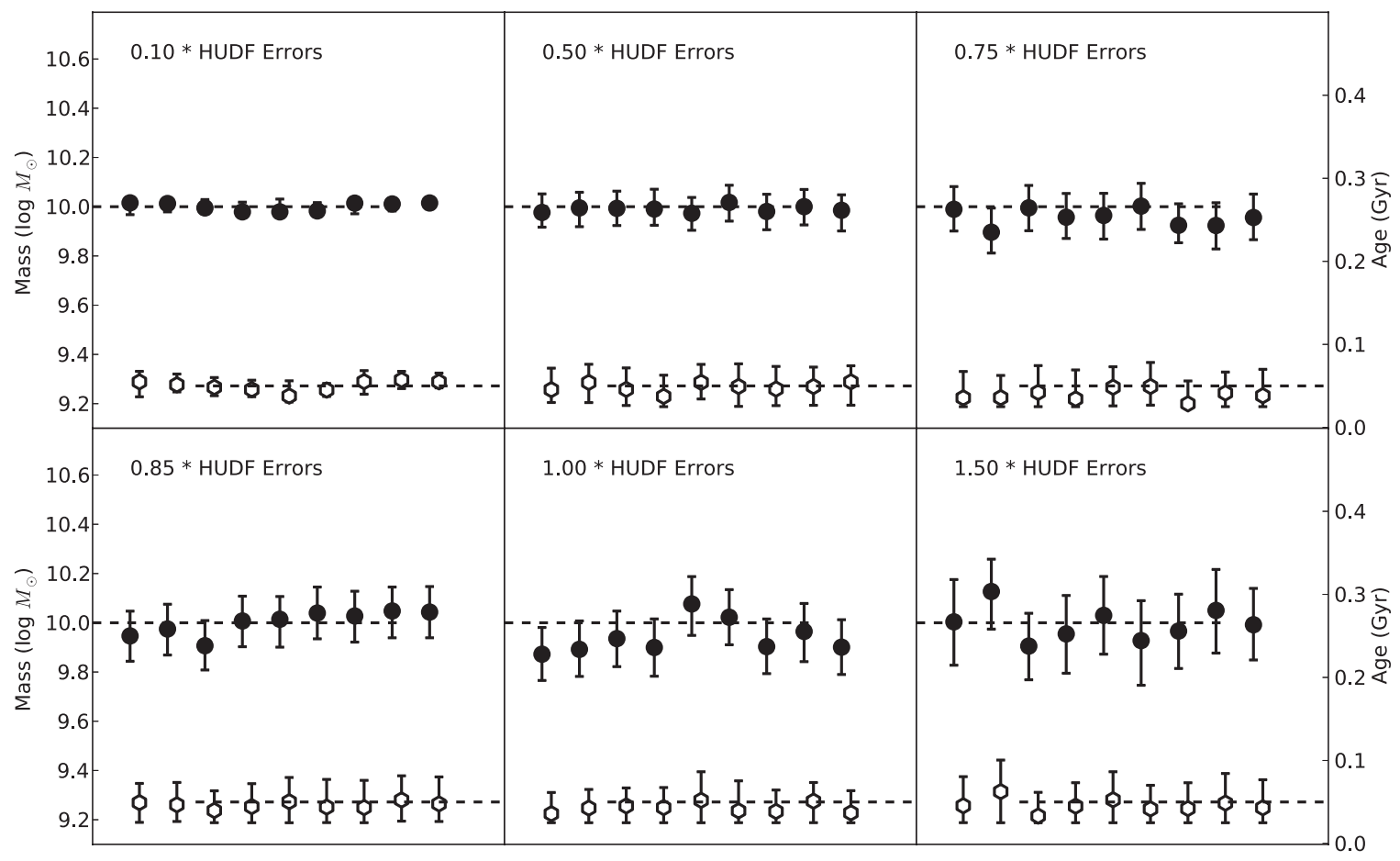

Figure 7. Same as Figure 6 but using input model that is a $10^{10} M_{\odot}$ galaxy with a single stellar population 50 Myr old and $A_{\mathrm{V}}=0.2$.

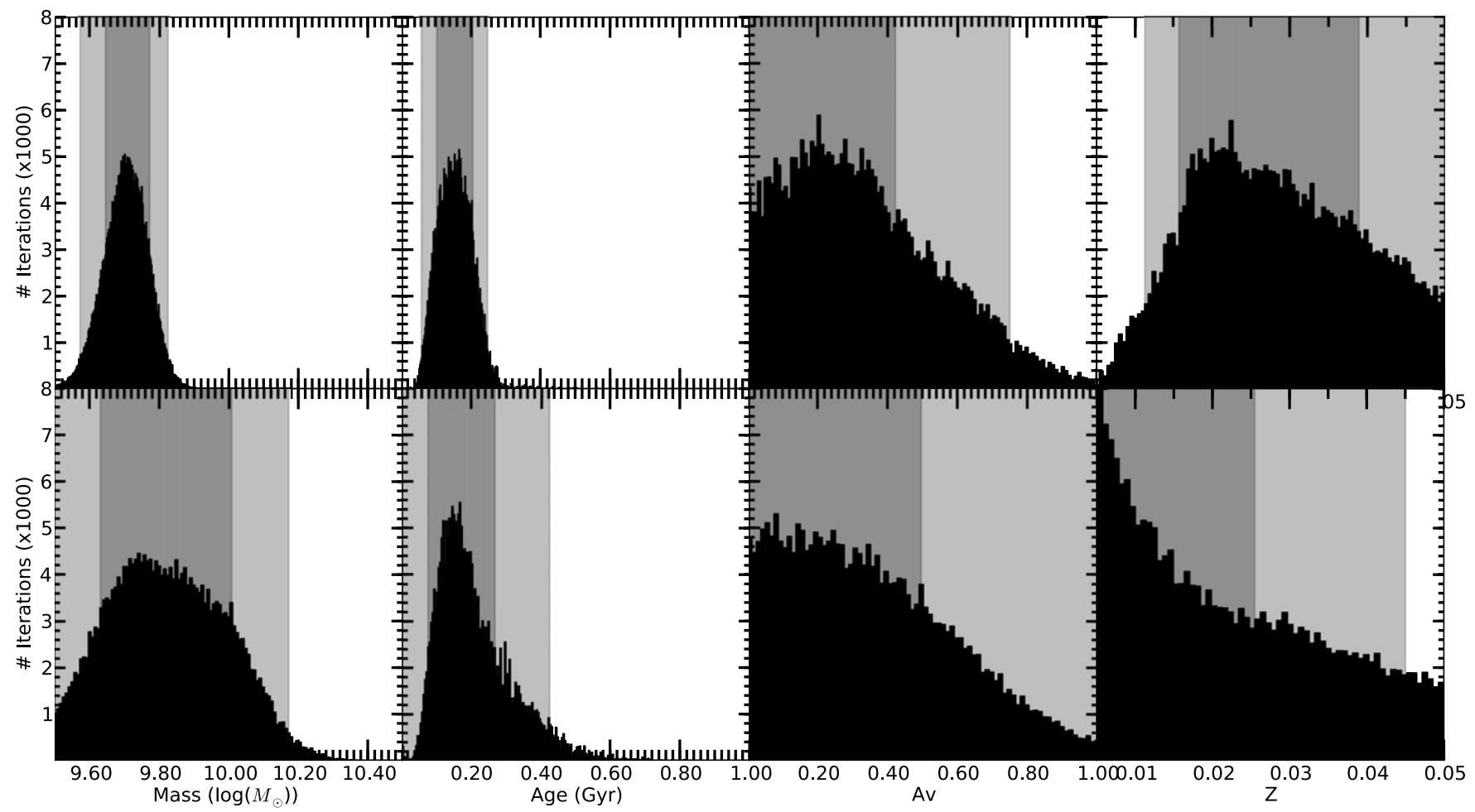

Figure 8. Credible regions for the stellar mass, ages, extinction, and metallicity for a simulated single population object at $z=4.75$ with (top row) and without (bottom row) IRAC observations probing the rest-frame optical light of this object.

break. At $z=4.5$, these IRAC bands correspond to $\approx 0.6 \mu \mathrm{m}$ and $\approx 0.8 \mu \mathrm{m}$. The main question we attempt to answer here is whether current IRAC observations provide enough information to constrain the stellar ages and extinction of these sources.

As a first example, we re-fit the single stellar population galaxy from Section 3.3.2 with a single stellar population of $0.6 \mathrm{Gyr}, A_{\mathrm{V}}=0.2$, and a mass of $10^{10} M_{\odot}$. We use realistic HUDF level photometric noise. We test the recovery of these parameters with and without the two IRAC channels. The HUDF error levels are $15 \%$ for IRAC1 and $20 \%$ for the IRAC2. The results are shown in Figure 8. The results do not differ significantly. The addition of the two IRAC channels only marginally improves the estimates of the stellar ages. With and without IRAC data yield ages of $0.67_{-0.25}^{0.19} \mathrm{Gyr}$ versus $0.64_{-0.32}^{0.24} \mathrm{Gyr}, A_{\mathrm{V}}$ of $0.12_{-0.12}^{0.29}$ versus $0.17_{-0.17}^{0.53}$, and total $\log$ (mass) of $10.0_{-0.12}^{0.11}$ versus $10.0_{-0.18}^{0.20}$. In this simple case, 
the IRAC measurements do help narrow our estimate of $A_{\mathrm{V}}$, separating the degeneracy between the red colors of the older stellar population and the effects of extinction. However, it does not improve constraints on the stellar ages.

Next, the addition of the two IRAC channels are tested when considering the case of two stellar populations (SSP2) using HUDF levels of photometric noise. $\pi \mathrm{MC}^{2}$ produces credible intervals for the ages that are very broad. Including the IRAC photometry produces credible intervals of $\log ($ mass $)=9.53_{-0.60}^{0.52}$, compared to $\log$ (mass) $=9.08_{-0.72}^{0.78}$ without them. This is only a marginal improvement. Our testing indicated that the only scenario which yields tangible improvements in the credible intervals occurs when the photometric errors in all bands are kept at 0.1.HUDF (i.e., $\approx 1 \%$ for ACS and WFC3 bands and better than $3 \%$ in the IRAC bands). This produces posterior credible intervals of $0.655_{-0.13}^{0.12} \mathrm{Gyr}$ and $0.04_{-0.00}^{0.01}$ Gyr for the old and young stellar population, respectively, a mass fraction of $99.13_{-1.69}^{0.16}, A_{\mathrm{V}}$ of $0.27_{-0.27}^{0.08}$, and a total stellar $\log$ (mass) of $10.05_{-0.14}^{0.06}$. However, it should be noted that, to date, such small photometric errors have yet to be achieved.

\subsection{Models and Redshifts}

Until now, we have restricted our simulations to a narrow range of redshifts because we have been interested in determining the physical properties of high-redshift galaxies for which detailed spectroscopic observations are difficult with current instrumentation. However, for $\pi \mathrm{MC}^{2}$ to be a robust technique, it should be capable of working within a much larger redshift space. We now apply $\pi \mathrm{MC}^{2}$ to a set of simulations with redshifts ranging from 1 to 8 . The simulated galaxy we use has a $0.1 \mathrm{Gyr}$ single stellar population, with an extinction of $A_{\mathrm{V}}=0.2$, and a mass of $10^{10} M_{\odot}$. In this section, we test how $\pi \mathrm{MC}^{2}$ deals with determining credible intervals for mass, age, and extinction using SSP and EXP stellar population models over a large redshift range. At each redshift, we have simulated the same object with random photometric noise added five separate times. We then used $\pi \mathrm{MC}^{2}$ to generate multiple MCMC chains, as outlined in Section 2.3.

Figures 9 and 10 show the results for the eight redshifts applied to the simulations. Within each redshift, we show the 95\% credible regions for the stellar mass, stellar age, or $A_{\mathrm{V}}$ when using an SSP model with the IRAC channels (left most shaded part of each redshift bin), an EXP model with the IRAC channels (darker shaded part of the redshift bins), and finally using an SSP model without the use of IRAC data. These figures also show the credible region median values using black circles. Figure 9 shows that while the mass is properly contained within the $95 \%$ SSP credible regions it does allow for lower mass solutions. The EXP models produce credible regions that are more centered on the fiducial values (shown using a solid line). The credible regions derived using an SSP model without the use of IRAC data show a widening of the credible region toward lower masses, especially for redshifts higher than 5 .

Figure 9 shows that the absence of IRAC data at $z>4$ results in a significant broadening of the credible regions we derive. Furthermore, this broadening is not symmetrical and the median values of the stellar masses and of the stellar ages are lower than the fiducial values. This is because at high redshifts the IRAC bands become more and more important for constraining the Balmer break. Beyond that, the number of filters which can be used decreases due to dropouts. This forces us to rely on fewer data points to constrain the models, allowing for a very wide range of stellar ages, masses, as well as of the other model parameters to become acceptable.

The credible regions for the extinction and metallicities for these simulations are shown in Figure 10. This figure further shows how metallicity is in most cases poorly constrained, which is something that we already saw earlier in this paper. We also see that the estimates of these parameters can be biased toward higher values as the redshift of the simulated sources is increased.

\section{APPLYING $\pi \mathrm{MC}^{2}$ TO OBSERVATIONS}

In this section, we now apply $\pi \mathrm{MC}^{2}$ to real observational data of high-redshift objects. For each object, we used the SSP, SSP2, EXP, Maximum $M / L$ SSP (Dickinson et al. 2003), and SPP+Nebular models and have derived credible regions for the stellar mass, stellar ages, and extinction for each of these objects. The SSP, SSP2, and EXP models were described earlier in this paper. The maximum $M / L$ SSP model is a variation of the two stellar population model (SSP2) where the older stellar population is fixed to have the maximum possible age (set to be the age of the universe) at the redshift of the source. These models are often used to determine the maximum fraction of an object's mass that can be in the form of an older stellar population.

\subsection{Observations}

Nine GRAPES sources are examined in this paper. The nine objects are taken from the GRAPES sources described in Pirzkal et al. (2007). As noted earlier, the GRAPES sample is based on spectroscopic detections of Ly $\alpha$ emission lines using the slitless grism mode of the ACS on HST. The nine objects were selected because they each have a clear $\operatorname{Ly} \alpha$ detection, which provides an independent confirmation of redshift. Since the GRAPES field nearly overlaps with the HUDF, there are many ancillary data available for these objects. These fields have been observed by nearly every modern observatory in the world. This allows us to constrain SEDs over a wide wavelength range. In Pirzkal et al. (2007), we relied on shallow NICMOS and Very Large Telescope observations to constrain the near-UV rest-frame luminosity and the rest-frame near UV slope. These data have since been supplemented by deep near-IR WFC3 observations. These new observations provide better constraints than the old NICMOS measurements. Using $\pi \mathrm{MC}^{2}$ combined with these new observations allows us to examine the nature of these objects in more detail. Table 1 lists the measured magnitudes of the Ly $\alpha$ sources that are detected in the 2009 WFC3 observations of the HUDF (ERS; GO-11359). Out of the nine sources listed in Pirzkal et al. (2007), two of them fall outside of the field of view of WFC3 (ID 631 and 712). In this paper, we take all other measurements of these sources in the observed optical and infrared bands (i.e., ACS and IRAC) from Pirzkal et al. (2007). The near-IR data are from the new WFC3 data. We use NICMOS measurements to supplement GRAPES sources not observed with WFC3. The WFC3 data reduction is described in McLure et al. (2009). The photometry was measured in the same manner and using the same detection maps as described in Pirzkal et al. (2007). The remaining sources discussed in this paper are taken from the literature and are a sample of 18 high- $z$ candidates, selected using the filter dropout method, supplemented by six $z>6$ gravitationally lensed candidates. The 18 sources at $z \approx 7$ and $z \approx 8$ are taken from Labbé et al. (2010) who stacked these observations prior to SED fitting to them. Here, we instead examine the individual sources that comprise the stacked data. 

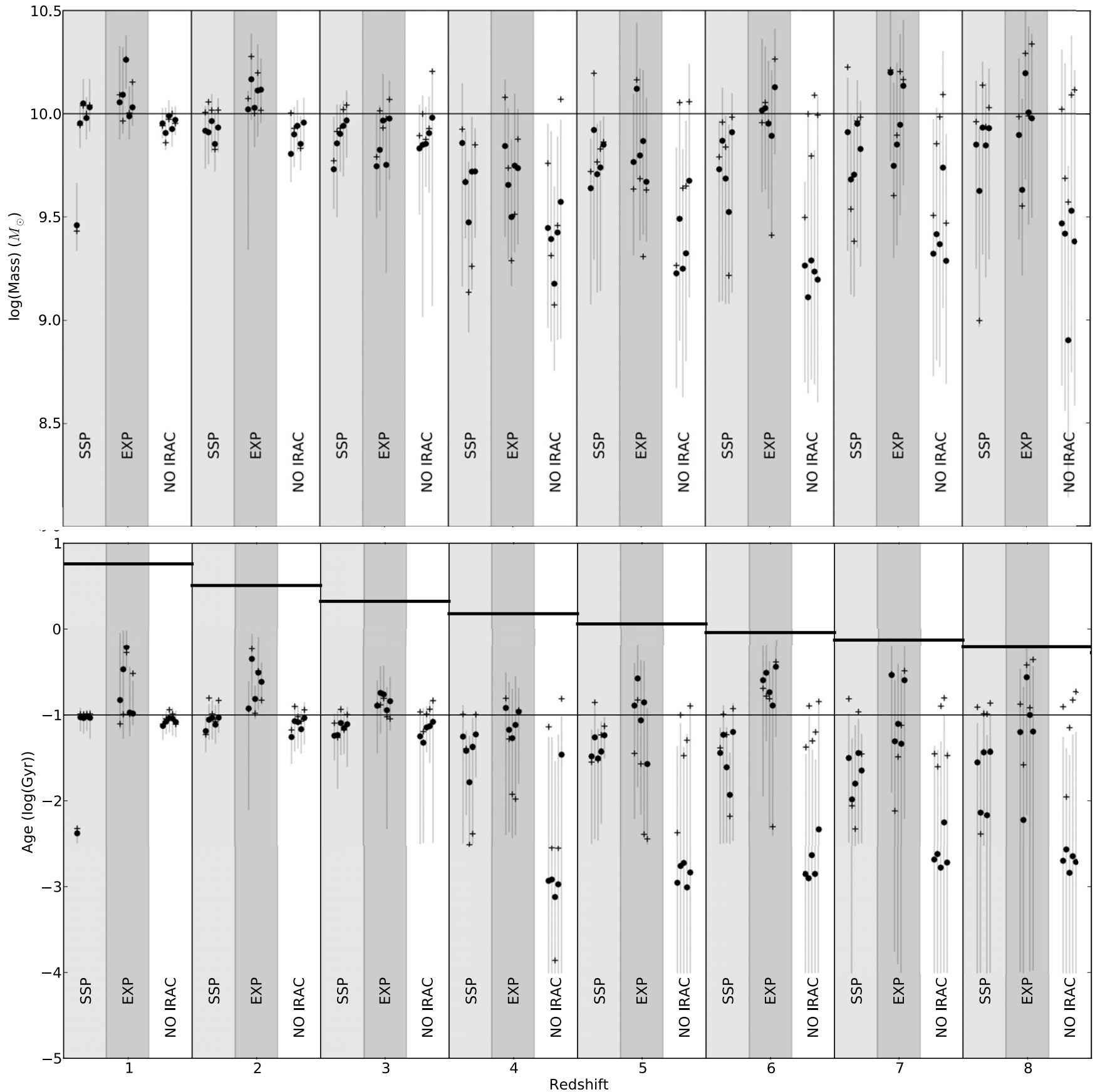

Figure 9. Recovered 95\% credible regions for the stellar mass (top panel) and stellar ages (bottom panel) when applying $\pi \mathrm{MC}^{2}$ to simulated observations of sources at redshifts ranging from $z=1$ to $z=8$. In each redshift band, we generated five random observations of each source, with realistic HUDF level photometric noise. We then use $\pi \mathrm{MC}^{2}$ using a single stellar population model (SSP), and exponentially decaying SFH model (EXP) and repeated the SSP model but excluding the IRAC bands. At each redshift, the three applications of $\pi \mathrm{MC}^{2}$ are shown with error bars. The best model fit values are shown with crosses. The median values of the derived credible intervals are shown using black circles. The true input model parameters $(\log (\operatorname{mass})=10$ and $\log (\operatorname{age})=-1)$ are shown using thin horizontal lines.

The remaining six lensed sources are high-redshift candidates lensed by nearby galaxy clusters and are taken from Bradley et al. (2008), Zheng et al. (2009), and Richard et al. (2011). Table 1 lists all the photometric measurements used in this paper. Individual measurements for the 18 Labbé et al. (2010) sources can be found in their Table 1.

\subsection{Results}

All of the results discussed in this section are plotted in Figures 11 and 12 and all of the credible regions we derive are listed in Tables 2-6.

\subsubsection{The GRAPES Sample}

The five models (SSP, SSP2, EXP, Maximum $M / L$ SSP, and SPP+Nebular) have been applied to all of the GRAPES sources and are shown in Figures 11 and 12. Figure 13 shows the onedimensional distributions of the model parameters for one of our sources (4442). Tables 2-5 list the $68 \%$ and $95 \%$ credible intervals for all of these sources. In all cases, the use of a double population model does not significantly improve the fit. It does neither yields significantly higher maximum stellar masses nor comes close to constraining the age of any possible 

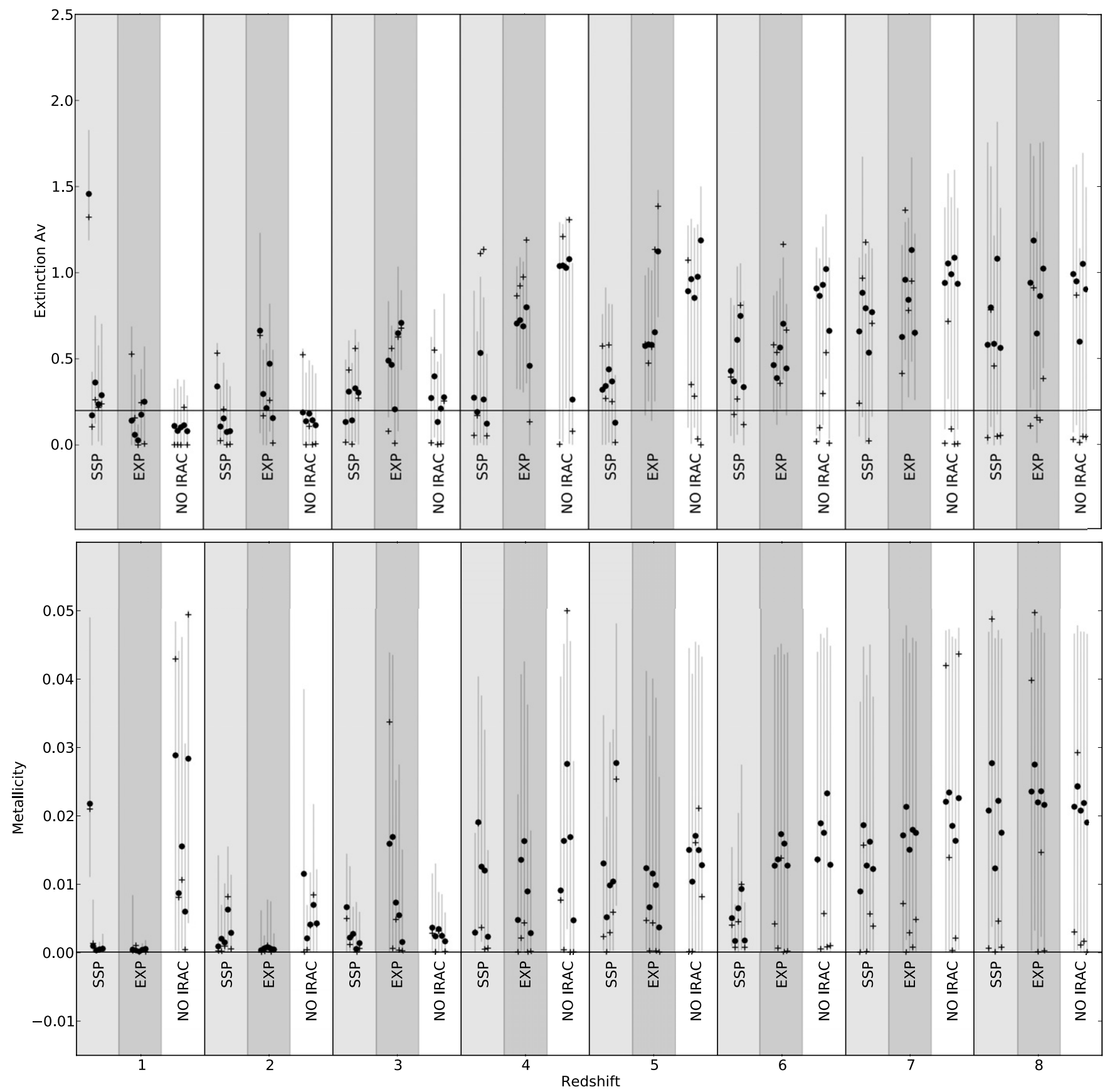

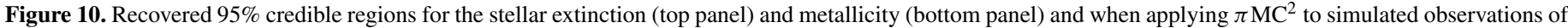

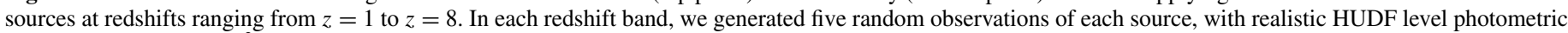

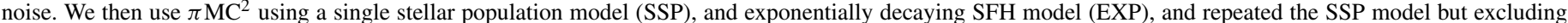

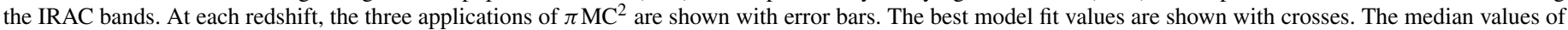
the derived credible intervals are shown using black circles. The true input model parameters $\left(A_{\mathrm{V}}=0.2\right.$ and $\left.Z=0.001\right)$ are shown using thin horizontal lines.

old stellar populations in these objects. As demonstrated earlier, constraining old stellar ages requires photometric precision beyond what has been achieved to date. However, we can conclude from Table 2 that these sources do appear to be less than 50 Myr old and the single population ages are constrained to be low. Table 2 lists the maximum formation redshifts of sources assuming an SSP population and maximum stellar population ages derived using the listed $95 \%$ credible intervals. All GRAPES sources could have formed at a redshift $z<6$. The allowed range of extinction values is somewhat broad, but we estimate that $A_{\mathrm{V}} \leqslant 1$ in these objects. Our results show that the masses are $10^{7}$ or $10^{8} M_{\odot}$, although they can vary by up to a factor of 20 , depending on the specific star formation history model we consider. These are sub- $M^{*}$ values (where $M^{*}=3 \times 10^{10} M_{\odot} ;$ Bell et al. 2003). In all cases, the addition of deep WFC3 near-IR photometry and the use of a more powerful SED-fitting technique have confirmed that the GRAPES object are consistent with a very young stellar population (Pirzkal et al. 2007). When fitting the GRAPES sample using an SSP model with nebular emission, we find that four of them $(4442,5183$, $9040,9340)$ appear to have $f_{\text {esc }}<0.5$ and that two sources $(631$, $6139)$ have $f_{\text {esc }}>0.5$. The rest $(712,5225,9487)$ have very broad ranges of acceptable values of $f_{\text {esc }}$. It should be noted that in this case $\pi \mathrm{MC}^{2}$ produces credible intervals that are not limited to 

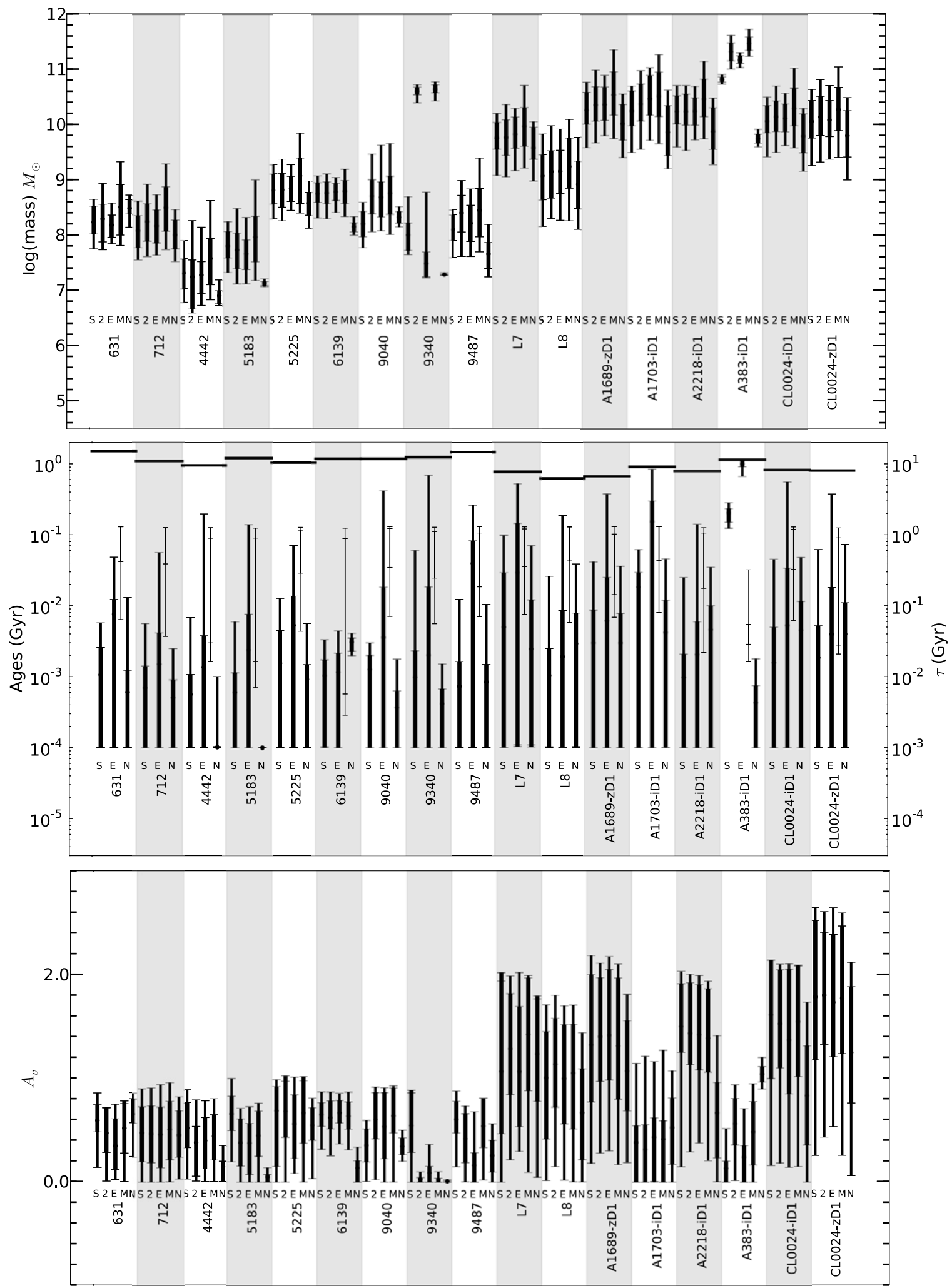

Figure 11. Top panel shows the median masses (black circles) and $68 \%$ and $95 \%$ credible regions (error bars) for all of the high- $z$ sources considered in Section 4 of this paper. The stellar population ages and the extinction credible regions are shown in the middle and bottom panels, respectively. For each source, from left to right, we show the results of the different models we considered. The SSP, SSP2, EXP, Max $M / L$, and SSP+Nebular results with S, 2, E, M, and N, respectively. In the middle panel, values of $\tau$ are shown with thin error bars.

either very low or very high $f_{\text {esc }}$ values. This is different from recent work by Schaerer et al. (2011) which used a non-MCMC method based on grid-based parameter fitting and concluded that $f_{\text {esc }}$ must either be negligible or close to unity.

\subsubsection{WFC3 HUDF Objects}

Recently, Labbé et al. (2010) have identified several possible high-redshift sources which the authors claim may represent 

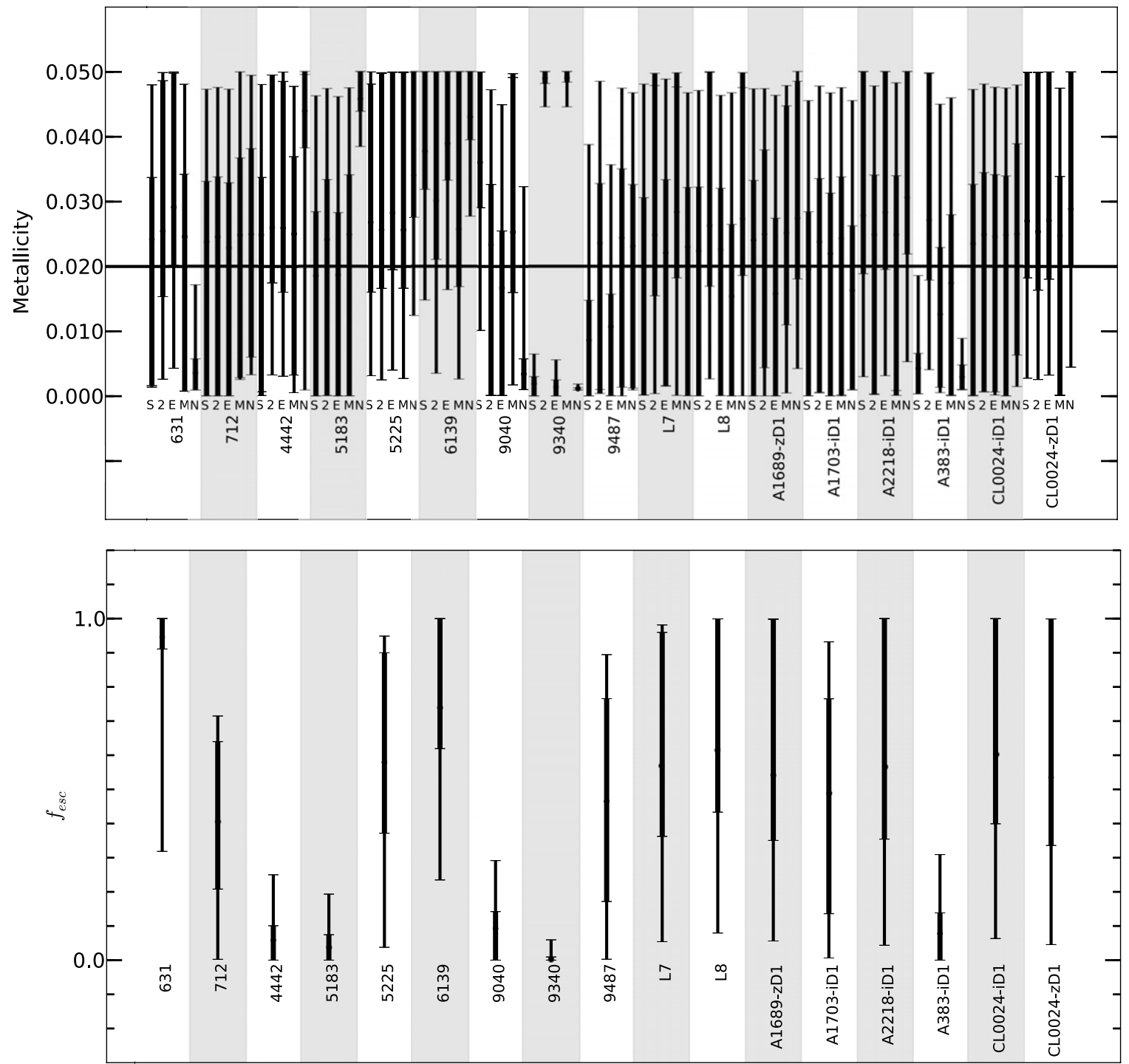

Figure 12. Same as Figure 11, but we now show the metallicity (top panel) and $f_{\text {esc }}$, the ionizing radiation escape fraction in the case of the SSP+Nebular mode (bottom panel).

the most distant objects identified to date in the universe. The redshifts are based on WFC3 F850LP and F098M dropouts noted in the WFC3 Early-Release Science observations of the HUDF. The SED fitting was carried out on stacked data of 36 putative $z \sim 7$ sources (data for 15 of which are given in their Table 1) and 3 putative $z \sim 8$ sources (all listed in their Table 1). The authors chose to stack the flux densities of the $z \sim 7$ sources in three $\sim 1$ mag bins and stack the flux densities of the three $z \sim 8$ sources. BC03 SEDs assuming a Salpeter Initial Mass Function (Salpeter 1955) from 0.1 to $100 M_{\odot}$ were then fit to the stacked objects (see their Figures 1 and 3) using a $\chi^{2}$ fitting technique. The best-fitting SEDs for the $z \sim 7$ objects yield $\log$ (mass) $\sim 9-9.8$ with $Z \sim 0.2-1$, with constant star formation (CSF) and a stellar age of 0.7 Gyr (see their Figure 1). The $z \sim 8$ stacked object yielded $\log$ (mass) $\sim 9.3$, $Z \sim 0.2$, with CSF and a stellar age of $0.3 \mathrm{Gyr}$. These results are particularly surprising, given that the age of the universe at these redshifts is $\sim 0.6$ Gyr. However, it is unclear what conclusions can be drawn from fitting stacked SEDs (as discussed in Nilsson et al. 2011).

The results from Labbé et al. (2010) provide an interesting test for the $\pi \mathrm{MC}^{2}$ method. We started by first assuming nothing about the redshift of these sources. Unlike the GRAPES sample, these objects have no spectroscopic observations that can be used to confirm their redshifts. Thus, redshift was left as a free parameter to test whether their SEDs might be consistent with low-redshift interlopers. Fluxes for the sources were taken from Table 1 of Labbé et al. (2010). We note that their Table 1 lists negative flux densities for some objects. In this case, and when detections were within $1 \sigma$ of the reported errors, we used upper limits for these bands. As noted earlier, upper limits to the flux are used as part of a penalized likelihood. We also note that their $Y$-band filter is actually a composite of fluxes from the F098M and F105W filters. For the putative $z \sim 7$ targets we applied $\pi \mathrm{MC}^{2}$ to the individual sources and not a stacked composite, as their Table 1 provides data for only 15/36 sources used to create their three stacked objects. We also applied $\pi \mathrm{MC}^{2}$ to the three $z \sim 8$ sources individually.

The results are shown in Figures 11-15. In Figures 11 and 12, we show the combined credible regions for the $z \approx 7$ and for the $z \approx 8$ sources. They are labeled L7 and L8. These were determined by combining the individual credible regions of the 15 sources at $z \sim 7$ (L7) and combining the three individual sources listed at $z \sim 8$ (L8). As shown in these two figures, 
Table 2

$\pi \mathrm{MC}^{2}$ SSP Results for Object Listed in Table 1

\begin{tabular}{|c|c|c|c|c|c|}
\hline UID & $\begin{array}{l}\text { Mass } \\
\left(M_{\odot}\right)\end{array}$ & $\begin{array}{l}\text { Age } \\
(\mathrm{Gyr})\end{array}$ & $A_{\mathrm{V}}^{\mathrm{a}}$ & $Z^{\mathrm{b}}$ & $z_{f}^{\mathrm{c}}$ \\
\hline 631 & $8.09_{-0.40,-0.31}^{+0.51,+0.24}$ & $0.003_{-0.003,-0.003}^{+0.003,0.001}$ & $0.49_{-0.49,-0.30}^{+0.27,+0.24}$ & $0.02_{-0.02,-0.02}^{+0.02,+0.01}$ & 4.0 \\
\hline 712 & $8.16_{-0.58,-0.34}^{+0.57,+0.30}$ & $0.001_{-0.001,-0.001}^{+0.008,0.001}$ & $0.52_{-0.52,-0.29}^{+0.45,+0.29}$ & $0.02_{-0.02,-0.02}^{+0.03,+0.01}$ & 5.2 \\
\hline 4442 & $\begin{array}{l}7.24_{-0.49,-0.29}^{+0.58,+0.24} \\
\text { a. }\end{array}$ & $0.001_{-0.000,-0.000}^{+0.006,0.001}$ & $0.45_{-0.43,-0.21}^{+0.38,+0.22}$ & $0.03_{-0.02,-0.01}^{+0.02,+0.02}$ & 5.8 \\
\hline 5183 & $7.58_{-0.57,-0.33}^{+0.50,+0.29}$ & $0.001_{-0.001,-0.001}^{+0.012,0.001}$ & $0.39_{-0.39,-0.39}^{+0.35,+0.12}$ & $0.02_{-0.02,-0.02}^{+0.03,+0.01}$ & 4.8 \\
\hline 5225 & $\begin{array}{l}8.75_{-0.49,-0.32}^{+0.49,+0.26} \\
\text {. }\end{array}$ & $0.005_{-0.004,-0.004}^{+0.010,0.002}$ & $0.37_{-0.37,-0.37}^{+0.61,+0.38}$ & $0.03_{-0.03,-0.01}^{+0.02,+0.02}$ & 5.5 \\
\hline 6139 & $8.76_{-0.42,-0.15}^{+0.31,+0.19}$ & $0.001_{-0.001,-0.001}^{+0.002,0.001}$ & $0.65_{-0.28,-0.09}^{+0.22,+0.13}$ & $0.04_{-0.02,-0.01}^{+0.01,+0.01}$ & 4.9 \\
\hline 9040 & $8.62_{-0.58,-0.24}^{+0.50,+0.29}$ & $0.001_{-0.001,-0.001}^{+0.010,0.001}$ & $0.65_{-0.58,-0.17}^{+0.34,+0.24}$ & $0.02_{-0.02,-0.02}^{+0.03,+0.01}$ & 4.9 \\
\hline 9340 & $7.47_{-0.41,-0.12}^{+0.43,+0.17}$ & $0.001_{-0.001,-0.001}^{+0.009,0.001}$ & $0.08_{-0.08,-0.08}^{+0.31,+0.07}$ & $0.00_{-0.00,-0.00}^{+0.00,+0.00}$ & 4.7 \\
\hline 9487 & $8.06_{-0.51,-0.26}^{+0.44,+0.27}$ & $0.001_{-0.001,-0.001}^{+0.019,0.002}$ & $0.55_{-0.55,-0.17}^{+0.25,+0.29}$ & $0.01_{-0.01,-0.01}^{+0.03,+0.00}$ & 4.1 \\
\hline L7-01 & $9.75_{-0.80,-0.27}^{+0.52,+0.34}$ & $0.010_{-0.010,-0.010}^{+0.089,0.015}$ & $1.21_{-1.11,-0.77}^{+1.21,+0.76}$ & $0.02_{-0.02,-0.02}^{+0.03,+0.01}$ & 7.8 \\
\hline L8-01 & $8.59_{-0.90,-0.55}^{+0.86,+0.57}$ & $0.001_{-0.001,-0.001}^{+0.027,0.002}$ & $0.59_{-0.59,-0.59}^{+0.97,+0.35}$ & $0.02_{-0.02,-0.02}^{+0.03,+0.01}$ & 8.3 \\
\hline A1689-zD1 & $10.26_{-0.68,-0.25}^{+0.51,+0.32}$ & $0.003_{-0.003,-0.003}^{+0.039,0.006}$ & $1.32_{-1.14,-0.55}^{+0.86,+0.68}$ & $0.02_{-0.02,-0.02}^{+0.02,+0.01}$ & 8.0 \\
\hline A1703-iD1 & $10.25_{-0.75,-0.27}^{+0.45,+0.36}$ & $0.018_{-0.018,-0.018}^{+0.044,0.011}$ & $0.38_{-0.38,-0.38}^{+0.76,+0.16}$ & $0.02_{-0.02,-0.02}^{+0.03,+0.01}$ & 6.3 \\
\hline A2218-iD1 & $10.24_{-0.64,-0.23}^{+0.46,+0.28}$ & $0.001_{-0.001,-0.001}^{+0.024,0.001}$ & $\begin{array}{l}1.50_{-1.35,-0.25}^{+0.53,+0.41} \\
\text { (1. }\end{array}$ & $0.03_{-0.02,-0.01}^{+0.02,+0.02}$ & 6.9 \\
\hline A383-iD1 & $10.81_{-0.08,-0.04}^{+0.09,+0.05}$ & $0.203_{-0.080,-0.052}^{+0.080,0.033}$ & $0.13_{-0.13,-0.13}^{+0.39,+0.07}$ & $0.00_{-0.00,-0.00}^{+0.01,+0.00}$ & 8.0 \\
\hline CL0024-iD1 & $10.06_{-0.65,-0.21}^{+0.44,+0.28}$ & $0.002_{-0.001,-0.001}^{+0.044,0.003}$ & $1.61_{-1.45,-0.62}^{+0.53,+0.53}$ & $0.02_{-0.02,-0.02}^{+0.02,+0.01}$ & 6.8 \\
\hline CL0024-zD1 & $10.05_{-0.80,-0.30}^{+0.58,+0.38}$ & $0.002_{-0.002,-0.002}^{+0.060,0.003}$ & $1.78_{-1.53,-0.61}^{+0.86,+0.74}$ & $0.03_{-0.02,-0.01}^{+0.02,+0.02}$ & 7.0 \\
\hline
\end{tabular}

Notes. This table lists the median values for each parameter as well as the $95 \%$ and $68 \%$ credible regions, separated by a comma.

a Extinction (Calzetti et al. 2000).

b Metallicity (Solar metallicity is $Z=0.02$ ).

${ }^{\mathrm{c}}$ Earliest formation redshift.

Table 3

$\pi \mathrm{MC}^{2}$ EXP Results for Object Listed in Table 1

\begin{tabular}{|c|c|c|c|c|c|}
\hline UID & $\begin{array}{l}\text { Mass } \\
\left(M_{\odot}\right)\end{array}$ & $\begin{array}{l}\text { Age } \\
(\mathrm{Gyr})\end{array}$ & $A_{\mathrm{V}}{ }^{\mathrm{a}}$ & $Z^{\mathrm{b}}$ & $\begin{array}{c}\tau \\
(\mathrm{Gyr})\end{array}$ \\
\hline 631 & $8.18_{-0.34,-0.22}^{+0.40,+0.18}$ & $0.008_{-0.008,-0.008}^{+0.041,0.005}$ & $0.34_{-0.32,-0.23}^{+0.40,+0.26}$ & $0.03_{-0.02,-0.01}^{+0.02,+0.02}$ & $\begin{array}{l}6.51_{-5.88,-2.33}^{+6.44,+6.47} \\
\end{array}$ \\
\hline 712 & $8.17_{-0.53,-0.31}^{+0.57,+0.29}$ & $0.002_{-0.001,-0.001}^{+0.055,0.003}$ & $0.46_{-0.46,-0.32}^{+0.48,+0.26}$ & $0.02_{-0.02,-0.02}^{+0.02,+0.01}$ & $\begin{array}{l}6.63_{-6.26,-2.75}^{+5.97,+5.95} \\
\end{array}$ \\
\hline 4442 & $7.27_{-0.55,-0.34}^{+0.87,+0.24}$ & $0.001_{-0.001,-0.001}^{+0.195,0.002}$ & $0.39_{-0.39,-0.27}^{+0.38,+0.22}$ & $0.03_{-0.02,-0.01}^{+0.02,+0.02}$ & $6.43_{-6.13,-6.26}^{+6.19,+2.52}$ \\
\hline 5183 & $7.66_{-0.54,-0.29}^{+0.66,+0.25}$ & $0.002_{-0.002,-0.002}^{+0.137,0.005}$ & $0.38_{-0.38,-0.30}^{+0.35,+0.19}$ & $0.02_{-0.02,-0.02}^{+0.03,+0.01}$ & $\begin{array}{l}6.45_{-6.38,-6.29}^{+5.96,+2.53} \\
\end{array}$ \\
\hline 5225 & $8.83_{-0.39,-0.23}^{+0.43,+0.22}$ & $0.005_{-0.005,-0.005}^{+0.065,0.008}$ & $0.56_{-0.48,-0.33}^{+0.45,+0.28}$ & $0.03_{-0.02,-0.01}^{+0.02,+0.02}$ & $\begin{array}{l}6.53_{-6.09,-3.65}^{+6.22,+5.11} \\
\text { to. }\end{array}$ \\
\hline 6139 & $8.78_{-0.37,-0.16}^{+0.26,+0.16}$ & $0.001_{-0.001,-0.001}^{+0.003,0.001}$ & $0.67_{-0.30,-0.10}^{+0.19,+0.12}$ & $0.04_{-0.02,-0.01}^{+0.01,+0.01}$ & $\begin{array}{c}6.47_{-6.41,-6.44}^{+5.92,+2.38} \\
\end{array}$ \\
\hline 9040 & $8.68_{-0.60,-0.35}^{+0.95,+0.28}$ & $0.004_{-0.003,-0.003}^{+0.413,0.015}$ & $0.53_{-0.53,-0.31}^{+0.38,+0.33}$ & $0.02_{-0.02,-0.02}^{+0.03,+0.01}$ & $6.54_{-5.83,-3.07}^{+6.44,+5.67}$ \\
\hline 9340 & $\begin{array}{c}7.49_{-0.26,-0.24}^{+1.29,+0.21} \\
\text { t. }\end{array}$ & $0.002_{-0.002,-0.002}^{+0.690,0.016}$ & $0.09_{-0.09,-0.09}^{+0.28,+0.06}$ & $0.00_{-0.00,-0.00}^{+0.00,+0.00}$ & $\begin{array}{l}6.52_{-5.96,-4.07}^{+6.27,+4.58} \\
\text { a }\end{array}$ \\
\hline 9487 & $8.21_{-0.61,-0.33}^{+0.62,+0.33}$ & $0.040_{-0.039,-0.039}^{+0.223,0.042}$ & $0.16_{-0.16,-0.16}^{+0.51,+0.12}$ & $0.01_{-0.01,-0.01}^{+0.02,+0.00}$ & $6.56_{-5.86,-4.71}^{+6.44,+4.03}$ \\
\hline L7-01 & $9.79_{-0.66,-0.33}^{+0.67,+0.35}$ & $0.046_{-0.046,-0.046}^{+0.571,0.109}$ & $1.28_{-0.97,-0.49}^{+0.97,+0.59}$ & $0.03_{-0.02,-0.01}^{+0.02,+0.02}$ & $\begin{array}{l}6.35_{-6.07,-3.45}^{+6.24,+5.28} \\
\text { t. }\end{array}$ \\
\hline L8-01 & $8.70_{-0.91,-0.47}^{+0.84,+0.54}$ & $0.002_{-0.002,-0.002}^{+0.212,0.007}$ & $0.70_{-0.70,-0.66}^{+0.87,+0.29}$ & $0.02_{-0.02,-0.02}^{+0.02,+0.01}$ & $\begin{array}{c}6.47_{-5.88,-5.90}^{+6.42,+2.92}\end{array}$ \\
\hline A1689-zD1 & $10.36_{-0.57,-0.29}^{+0.53,+0.31}$ & $0.006_{-0.006,-0.006}^{+0.372,0.019}$ & $1.41_{-1.11,-0.43}^{+0.76,+0.63}$ & $0.02_{-0.02,-0.02}^{+0.03,+0.01}$ & $\begin{array}{l}6.50_{-5.81,-5.06}^{+6.49,+3.72} \\
\text { t. }\end{array}$ \\
\hline A1703-iD1 & $10.46_{-0.75,-0.30}^{+0.57,+0.43}$ & $0.154_{-0.154,-0.154}^{+0.689,0.147}$ & $0.43_{-0.43,-0.43}^{+0.73,+0.19}$ & $0.02_{-0.02,-0.02}^{+0.02,+0.01}$ & $6.61_{-5.80,-2.29}^{+6.39,+6.39}$ \\
\hline A2218-iD1 & $10.24_{-0.52,-0.25}^{+0.45,+0.24}$ & $0.002_{-0.002,-0.002}^{+0.139,0.004}$ & $1.42_{-1.04,-0.34}^{+0.57,+0.48}$ & $0.03_{-0.03,-0.01}^{+0.02,+0.02}$ & $\begin{array}{c}6.47_{-6.25,-4.71}^{+6.09,+4.10} \\
\text { t. }\end{array}$ \\
\hline A383-iD1 & $11.17_{-0.14,-0.06}^{+0.13,+0.08}$ & $0.991_{-0.326,-0.079}^{+0.158,0.158}$ & $0.24_{-0.24,-0.24}^{+0.47,+0.11}$ & $0.01_{-0.01,-0.01}^{+0.03,+0.01}$ & $0.44_{-0.28,-0.16}^{+2.76,+0.10}$ \\
\hline CL0024-iD1 & $10.11_{-0.49,-0.24}^{+0.45,+0.26}$ & $0.005_{-0.005,-0.005}^{+0.552,0.029}$ & $1.37_{-1.23,-0.52}^{+0.73,+0.68}$ & $0.02_{-0.02,-0.02}^{+0.02,+0.01}$ & $\begin{array}{l}6.61_{-6.00,-3.37}^{+6.29,+5.32} \\
\text { t. }\end{array}$ \\
\hline CL0024-zD1 & $10.08_{-0.71,-0.30}^{+0.62,+0.35}$ & $0.004_{-0.004,-0.004}^{+0.371,0.014}$ & $1.73_{-1.20,-0.53}^{+0.91,+0.65}$ & $0.03_{-0.02,-0.01}^{+0.02,+0.02}$ & $6.44_{-6.23,-6.16}^{+6.06,+2.59}$ \\
\hline
\end{tabular}

Notes. This table lists the median values for each parameter as well as the $95 \%$ and $68 \%$ credible regions, separated by a comma.

${ }^{a}$ Extinction (Calzetti et al. 2000).

b Metallicity (Solar metallicity is $Z=0.02$ ).

the combined credible regions indicate that these objects, as a whole, have stellar masses marginally larger than those of the GRAPES sample and have potentially much larger values of extinction. The range of acceptable metallicities and $f_{\text {esc }}$ are large and relatively unconstrained. Given the broad composite credible regions and the absence of any evidence suggesting 
Table 4

$\pi \mathrm{MC}^{2}$ SSP2 Results for Object Listed in Table 1

\begin{tabular}{|c|c|c|c|c|c|c|c|}
\hline UID & $\begin{array}{l}\text { Mass } \\
\left(M_{\odot}\right)\end{array}$ & $\begin{array}{l}\text { Age }_{\text {old }} \\
\text { (Gyr) }\end{array}$ & $A_{\mathrm{V}^{\mathrm{a}}}^{\mathrm{a}}$ & $Z_{\text {old }}^{\text {b }}$ & $\%$ Old & $\begin{array}{l}\text { Age Young } \\
\text { (Gyr) }\end{array}$ & $Z_{\text {young }}{ }^{\mathrm{b}}$ \\
\hline 631 & $\begin{array}{c}8.29_{-0.56,-0.42}^{+0.65,+0.27} \\
\end{array}$ & $0.045_{-0.045,-0.045}^{+1.026,0.108}$ & $0.47_{-0.46,-0.19}^{+0.25,+0.24}$ & $0.03_{-0.02,-0.01}^{+0.02,+0.02}$ & $44.69_{-44.61,-44.59}^{+43.55,+16.40}$ & $0.001_{-0.001,-0.001}^{+0.004,0.001}$ & $0.03_{-0.02,-0.01}^{+0.02,+0.02}$ \\
\hline 712 & $8.25_{-0.63,-0.36}^{+0.67,+0.32}$ & $\begin{array}{l}0.029_{-0.029,-0.029}^{+0.786,0.087} \\
\end{array}$ & $0.46_{-0.46,-0.28}^{+0.44,+0.27}$ & $0.02_{-0.02,-0.02}^{+0.02,+0.01}$ & $\begin{array}{l}42.68_{-42.68,-42.68}^{+47.34,+17.48} \\
\end{array}$ & $0.001_{-0.000,-0.000}^{+0.004,0.001}$ & $0.02_{-0.02,-0.02}^{+0.02,+0.01}$ \\
\hline 4442 & $7.24_{-0.65,-0.58}^{+1.02,+0.31}$ & $0.043_{-0.042,-0.042}^{+0.628,0.079}$ & $0.38_{-0.37,-0.32}^{+0.41,+0.16}$ & $0.03_{-0.02,-0.01}^{+0.02,+0.02}$ & $56.26_{-50.36,-19.56}^{+42.71,+42.34}$ & $0.001_{-0.001,-0.001}^{+0.015,0.001}$ & $0.03_{-0.02,-0.01}^{+0.02,+0.02}$ \\
\hline 5183 & $7.73_{-0.62,-0.34}^{+0.75,+0.30}$ & $0.033_{-0.033,-0.033}^{+0.840,0.092}$ & $0.38_{-0.38,-0.23}^{+0.33,+0.23}$ & $0.02_{-0.02,-0.02}^{+0.02,+0.01}$ & $46.87_{-46.87,-46.87}^{+45.54,+17.90}$ & $0.001_{-0.001,-0.001}^{+0.006,0.001}$ & $0.02_{-0.02,-0.02}^{+0.03,+0.01}$ \\
\hline 5225 & $8.82_{-0.56,-0.32}^{+0.55,+0.30}$ & $0.046_{-0.045,-0.045}^{+0.737,0.098}$ & $0.68_{-0.68,-0.25}^{+0.29,+0.34}$ & $0.03_{-0.02,-0.01}^{+0.02,+0.02}$ & $36.48_{-36.48,-36.48}^{+51.59,+19.68}$ & $0.001_{-0.001,-0.001}^{+0.009,0.001}$ & $0.03_{-0.03,-0.01}^{+0.02,+0.02}$ \\
\hline 6139 & $8.76_{-0.47,-0.19}^{+0.35,+0.20}$ & $0.008_{-0.008,-0.008}^{+0.887,0.090}$ & $0.62_{-0.37,-0.11}^{+0.25,+0.16}$ & $0.03_{-0.03,-0.01}^{+0.02,+0.02}$ & $31.47_{-31.47,-31.47}^{+58.81,+17.41}$ & $0.001_{-0.001,-0.001}^{+0.002,0.001}$ & $0.04_{-0.03,-0.01}^{+0.01,+0.01}$ \\
\hline 9040 & $8.71_{-0.65,-0.32}^{+0.76,+0.29}$ & $0.034_{-0.034,-0.034}^{+0.811,0.092}$ & $0.60_{-0.58,-0.19}^{+0.31,+0.26}$ & $0.02_{-0.02,-0.02}^{+0.02,+0.01}$ & $37.18_{-37.18,-37.18}^{+55.03,+21.66}$ & $0.001_{-0.000,-0.000}^{+0.009,0.001}$ & $0.02_{-0.02,-0.02}^{+0.03,+0.01}$ \\
\hline 9340 & $10.60_{-0.21,-0.07}^{+0.12,+0.07}$ & $\begin{array}{l}1.220_{-0.141,-0.033}^{+0.050,0.050} \\
\text { la }\end{array}$ & $0.03_{-0.03,-0.03}^{+0.07,+0.01}$ & $0.05_{-0.00,-0.00}^{+0.00,+0.00}$ & $99.95_{-0.04,-0.01}^{+0.00,+0.01}$ & $0.001_{-0.000,-0.000}^{+0.002,0.000}$ & $\begin{array}{l}0.00_{-0.00,-0.00}^{+0.00,+0.00} \\
\text { a. }\end{array}$ \\
\hline 9487 & $8.40_{-0.79,-0.35}^{+0.58,+0.32}$ & $0.068_{-0.068,-0.068}^{+0.985,0.114}$ & $0.42_{-0.42,-0.23}^{+0.31,+0.24}$ & $0.02_{-0.02,-0.02}^{+0.02,+0.01}$ & $55.88_{-51.33,-22.10}^{+40.01,+40.01}$ & $0.000_{-0.000,-0.000}^{+0.002,0.000}$ & $0.01_{-0.01,-0.01}^{+0.03,+0.01}$ \\
\hline L7-01 & $9.83_{-0.77,-0.32}^{+0.60,+0.34}$ & $0.080_{-0.080,-0.080}^{+0.509,0.090}$ & $1.39_{-1.33,-0.71}^{+0.92,+0.65}$ & $0.02_{-0.02,-0.01}^{+0.02,+0.02}$ & $53.06_{-53.03,-23.79}^{+40.35,+40.81}$ & $0.003_{-0.003,-0.003}^{+0.068,0.009}$ & $0.02_{-0.02,-0.02}^{+0.02,+0.01}$ \\
\hline L8-01 & $8.74_{-0.94,-0.49}^{+0.84,+0.55}$ & $0.026_{-0.026,-0.026}^{+0.423,0.060}$ & $0.70_{-0.70,-0.62}^{+0.80,+0.37}$ & $0.03_{-0.03,-0.03}^{+0.02,+0.01}$ & $45.37_{-44.96,-44.03}^{+47.53,+19.44}$ & $0.001_{-0.000,-0.000}^{+0.012,0.001}$ & $0.02_{-0.02,-0.02}^{+0.02,+0.01}$ \\
\hline A1689-zD1 & $\begin{array}{c}10.35_{-0.69,-0.29}^{+0.63,+0.33} \\
\text {. }\end{array}$ & $0.043_{-0.043,-0.043}^{+0.654,0.090}$ & $1.40_{-1.12,-0.43}^{+0.71,+0.57}$ & $0.02_{-0.02,-0.02}^{+0.02,+0.01}$ & $47.71_{-47.66,-47.68}^{+45.93,+17.46}$ & $0.001_{-0.001,-0.001}^{+0.022,0.002}$ & $0.02_{-0.02,-0.02}^{+0.02,+0.01}$ \\
\hline A1703-iD1 & $10.37_{-0.82,-0.31}^{+0.60,+0.36}$ & $0.109_{-0.109,-0.109}^{+0.781,0.129}$ & $0.31_{-0.31,-0.31}^{+0.90,+0.24}$ & $0.02_{-0.02,-0.02}^{+0.02,+0.01}$ & $59.62_{-53.59,-20.13}^{+40.05,+40.23}$ & $\begin{array}{l}0.010_{-0.010,-0.010}^{+0.035,0.008} \\
\end{array}$ & $0.02_{-0.02,-0.02}^{+0.02,+0.01}$ \\
\hline A2218-iD1 & $10.24_{-0.71,-0.25}^{+0.47,+0.31}$ & $\begin{array}{l}0.046_{-0.046,-0.046}^{+0.798,0.106} \\
\end{array}$ & $1.43_{-1.14,-0.30}^{+0.57,+0.49}$ & $0.02_{-0.02,-0.02}^{+0.02,+0.01}$ & $\begin{array}{r}1.00_{-1.00,-1.00}^{+67.18,+5.33} \\
\end{array}$ & $0.001_{-0.001,-0.001}^{+0.014,0.001}$ & $0.03_{-0.02,-0.01}^{+0.02,+0.02}$ \\
\hline A383-iD1 & $11.32_{-0.31,-0.16}^{+0.30,+0.16}$ & $0.458_{-0.307,-0.225}^{+0.498,0.157}$ & $0.56_{-0.55,-0.21}^{+0.37,+0.24}$ & $0.03_{-0.02,-0.01}^{+0.02,+0.02}$ & $99.15_{-2.62,-0.30}^{+0.57,+0.37}$ & $0.000_{-0.000,-0.000}^{+0.011,0.000}$ & $0.01_{-0.01,-0.01}^{+0.03,+0.01}$ \\
\hline CL0024-iD1 & $10.14_{-0.64,-0.27}^{+0.55,+0.28}$ & $0.048_{-0.048,-0.048}^{+0.829,0.112}$ & $1.53_{-1.34,-0.43}^{+0.57,+0.52}$ & $0.02_{-0.02,-0.02}^{+0.02,+0.01}$ & $44.99_{-44.99,-44.99}^{+48.91,+17.91}$ & $0.001_{-0.001,-0.001}^{+0.023,0.001}$ & $0.02_{-0.02,-0.02}^{+0.02,+0.01}$ \\
\hline CL0024-zD1 & $10.13_{-0.82,-0.33}^{+0.68,+0.37}$ & $0.040_{-0.040,-0.040}^{+0.801,0.103}$ & $1.80_{-1.37,-0.47}^{+0.81,+0.61}$ & $0.03_{-0.02,-0.01}^{+0.02,+0.02}$ & $44.44_{-44.44,-44.44}^{+48.11,+17.67}$ & $0.001_{-0.001,-0.001}^{+0.025,0.001}$ & $0.03_{-0.03,-0.01}^{+0.02,+0.02}$ \\
\hline
\end{tabular}

Notes. This table lists the median values for each parameter as well as the $95 \%$ and $68 \%$ credible regions, separated by a comma.

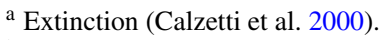

b Metallicity (Solar metallicity is $Z=0.02$ ).

Table 5

$\pi \mathrm{MC}^{2}$ Maximum $M / L$ SSP2 Results for Object Listed in Table 1

\begin{tabular}{|c|c|c|c|c|c|c|}
\hline UID & $\begin{array}{l}\text { Mass } \\
\left(M_{\odot}\right)\end{array}$ & $A_{\mathrm{V}}{ }^{\mathrm{a}}$ & $Z_{\text {old }}{ }^{\mathrm{b}}$ & $\%$ Old & $\begin{array}{c}\text { Age Young } \\
\text { (Gyr) }\end{array}$ & $Z_{\text {young }}{ }^{\mathrm{b}}$ \\
\hline 631 & $8.50_{-0.69,-0.51}^{+0.82,+0.40}$ & $0.51_{-0.51,-0.24}^{+0.24,+0.26}$ & $0.02_{-0.02,-0.02}^{+0.02,+0.01}$ & $54.60_{-49.73,-19.46}^{+41.39,+41.38}$ & $0.001_{-0.001,-0.001}^{+0.004,0.002}$ & $0.03_{-0.02,-0.02}^{+0.02,+0.02}$ \\
\hline 712 & $8.50_{-0.75,-0.42}^{+0.79,+0.38}$ & $0.51_{-0.51,-0.29}^{+0.45,+0.27}$ & $0.02_{-0.02,-0.02}^{+0.03,+0.01}$ & $45.14_{-45.13,-45.01}^{+44.24,+16.84}$ & $0.001_{-0.001,-0.001}^{+0.008,0.001}$ & $0.01_{-0.01,-0.01}^{+0.03,+0.01}$ \\
\hline 4442 & $7.57_{-0.75,-0.48}^{+1.05,+0.36}$ & $0.44_{-0.44,-0.23}^{+0.36,+0.21}$ & $0.03_{-0.02,-0.02}^{+0.02,+0.01}$ & $48.23_{-48.23,-48.23}^{+45.70,+18.04}$ & $0.001_{-0.000,-0.000}^{+0.005,0.001}$ & $0.03_{-0.02,-0.01}^{+0.02,+0.02}$ \\
\hline 5183 & $7.97_{-0.78,-0.45}^{+1.04,+0.38}$ & $0.45_{-0.45,-0.20}^{+0.31,+0.23}$ & $0.02_{-0.02,-0.02}^{+0.02,+0.01}$ & $48.16_{-48.16,-48.16}^{+46.35,+18.17}$ & $0.001_{-0.001,-0.001}^{+0.009,0.001}$ & $0.02_{-0.02,-0.02}^{+0.03,+0.01}$ \\
\hline 5225 & $9.07_{-0.67,-0.50}^{+0.77,+0.32}$ & $0.67_{-0.67,-0.29}^{+0.31,+0.35}$ & $0.03_{-0.02,-0.01}^{+0.02,+0.02}$ & $47.41_{-47.20,-36.20}^{+42.97,+28.09}$ & $0.001_{-0.001,-0.001}^{+0.008,0.002}$ & $0.03_{-0.03,-0.01}^{+0.02,+0.02}$ \\
\hline 6139 & $8.79_{-0.46,-0.22}^{+0.40,+0.20}$ & $0.64_{-0.32,-0.12}^{+0.23,+0.14}$ & $0.03_{-0.02,-0.01}^{+0.02,+0.02}$ & $14.27_{-14.27,-14.27}^{+51.70,+15.94}$ & $0.001_{-0.001,-0.001}^{+0.002,0.001}$ & $0.04_{-0.02,-0.01}^{+0.01,+0.01}$ \\
\hline 9040 & $8.76_{-0.75,-0.38}^{+0.90,+0.31}$ & $0.64_{-0.64,-0.17}^{+0.29,+0.27}$ & $0.03_{-0.02,-0.01}^{+0.02,+0.02}$ & $29.35_{-29.35,-29.35}^{+61.96,+24.20}$ & $0.001_{-0.001,-0.001}^{+0.012,0.001}$ & $0.02_{-0.02,-0.02}^{+0.03,+0.01}$ \\
\hline 9340 & $10.64_{-0.22,-0.07}^{+0.14,+0.08}$ & $0.02_{-0.02,-0.02}^{+0.08,+0.02}$ & $0.05_{-0.00,-0.00}^{+0.00,+0.00}$ & $99.95_{-0.02,-0.00}^{+0.02,+0.01}$ & $0.001_{-0.000,-0.000}^{+0.002,0.000}$ & $0.00_{-0.00,-0.00}^{+0.00,+0.00}$ \\
\hline 9487 & $8.44_{-0.75,-0.48}^{+0.95,+0.39}$ & $0.54_{-0.52,-0.21}^{+0.27,+0.27}$ & $0.02_{-0.02,-0.02}^{+0.02,+0.01}$ & $52.75_{-48.37,-20.32}^{+44.43,+44.06}$ & $0.001_{-0.001,-0.001}^{+0.019,0.003}$ & $0.01_{-0.01,-0.01}^{+0.03,+0.00}$ \\
\hline L7-01 & $9.99_{-0.86,-0.31}^{+0.60,+0.36}$ & $1.06_{-1.05,-1.00}^{+1.18,+0.39}$ & $0.03_{-0.03,-0.01}^{+0.02,+0.02}$ & $43.27_{-43.25,-43.25}^{+47.42,+16.55}$ & $0.012_{-0.012,-0.012}^{+0.076,0.018}$ & $0.02_{-0.02,-0.02}^{+0.03,+0.01}$ \\
\hline L8-01 & $8.66_{-0.93,-0.47}^{+0.75,+0.53}$ & $0.76_{-0.75,-0.63}^{+0.83,+0.41}$ & $0.03_{-0.02,-0.02}^{+0.02,+0.01}$ & $0.11_{-0.11,-0.11}^{+0.82,+0.11}$ & $0.001_{-0.001,-0.001}^{+0.021,0.001}$ & $0.02_{-0.02,-0.02}^{+0.02,+0.01}$ \\
\hline A1689-zD1 & $10.54_{-0.79,-0.37}^{+0.82,+0.42}$ & $1.32_{-1.14,-0.52}^{+0.78,+0.65}$ & $0.03_{-0.02,-0.01}^{+0.02,+0.02}$ & $48.19_{-48.13,-36.78}^{+44.97,+29.13}$ & $0.002_{-0.002,-0.002}^{+0.033,0.004}$ & $0.02_{-0.02,-0.02}^{+0.02,+0.01}$ \\
\hline A1703-iD1 & $10.52_{-0.86,-0.37}^{+0.75,+0.43}$ & $0.41_{-0.41,-0.41}^{+0.86,+0.18}$ & $0.02_{-0.02,-0.02}^{+0.02,+0.01}$ & $51.71_{-47.56,-20.92}^{+46.05,+44.77}$ & $0.012_{-0.012,-0.012}^{+0.033,0.007}$ & $0.02_{-0.02,-0.02}^{+0.03,+0.01}$ \\
\hline A2218-iD1 & $10.46_{-0.71,-0.32}^{+0.69,+0.36}$ & $\begin{array}{c}1.39_{-1.18,-0.33}^{+0.55,+0.48} \\
-1.0 .0\end{array}$ & $0.02_{-0.02,-0.02}^{+0.02,+0.01}$ & $42.59_{-42.59,-42.59}^{+46.42,+17.00}$ & $0.001_{-0.001,-0.001}^{+0.016,0.001}$ & $0.03_{-0.02,-0.01}^{+0.02,+0.02}$ \\
\hline A383-iD1 & $11.47_{-0.23,-0.13}^{+0.25,+0.11}$ & $0.48_{-0.48,-0.32}^{+0.46,+0.29}$ & $0.02_{-0.02,-0.02}^{+0.03,+0.01}$ & $99.34_{-2.28,-0.24}^{+0.47,+0.20}$ & $0.001_{-0.001,-0.001}^{+0.023,0.006}$ & $0.01_{-0.01,-0.01}^{+0.03,+0.01}$ \\
\hline CL0024-iD1 & $10.29_{-0.71,-0.31}^{+0.73,+0.37}$ & $\begin{array}{c}1.55_{-1.39,-0.53}^{+0.54,+0.54} \\
\text { c. }\end{array}$ & $0.02_{-0.02,-0.02}^{+0.02,+0.01}$ & $44.00_{-44.00,-44.00}^{+47.07,+17.71}$ & $0.002_{-0.001,-0.001}^{+0.032,0.003}$ & $0.02_{-0.02,-0.02}^{+0.02,+0.01}$ \\
\hline CL0024-zD1 & $10.27_{-0.87,-0.38}^{+0.77,+0.40}$ & $1.77_{-1.52,-0.54}^{+0.82,+0.70}$ & $0.02_{-0.02,-0.02}^{+0.02,+0.01}$ & $34.14_{-34.14,-34.14}^{+54.14,+19.25}$ & $0.002_{-0.002,-0.002}^{+0.049,0.002}$ & $0.03_{-0.03,-0.01}^{+0.02,+0.02}$ \\
\hline
\end{tabular}

Notes. This table lists the median values for each parameter as well as the $95 \%$ and $68 \%$ credible regions, separated by a comma.

${ }^{a}$ Extinction (Calzetti et al. 2000).

${ }^{\mathrm{b}}$ Metallicity (Solar metallicity is $Z=0.02$ ).

these objects are similar to each other, it is worth examining them individually. Figures 14 and 15 show the individual credible regions of the $z \approx 7$ and $z \approx 8$ sources. We have labeled these L7-01 through L7-15 and L8-1 through L8-3, respectively (corresponding to the objects from the top to the bottom of Table 1 in Labbé et al. 2010). These sources show a significant variation in stellar mass. The masses within the group vary by as much as 100 , with some objects in the $10^{8} M_{\odot}$ range, compared 

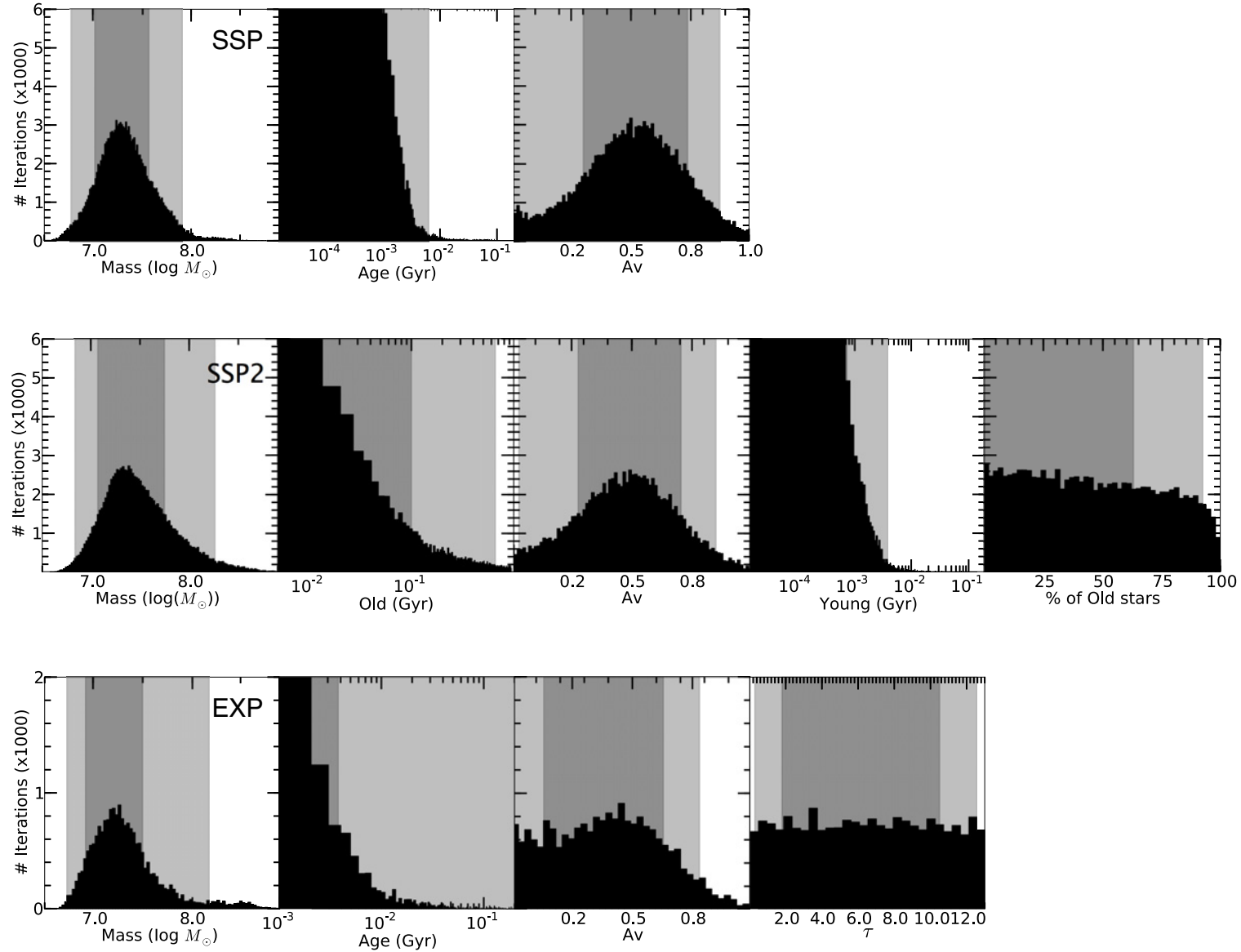

Figure 13. Posterior probability densities of the model parameters of the SSP, SSP2, and EXP models when applied to the GRAPES 4442 object.

Table 6

$\pi \mathrm{MC}^{2} \mathrm{SSP}+$ Nebular Results for Object Listed in Table 1

\begin{tabular}{|c|c|c|c|c|c|c|}
\hline UID & $\begin{array}{l}\text { Mass } \\
\left(M_{\odot}\right)\end{array}$ & $\begin{array}{l}\text { Age } \\
\text { (Gyr) }\end{array}$ & $A_{\mathrm{V}^{\mathrm{a}}}$ & $Z^{\mathrm{b}}$ & $f_{\mathrm{esc}}{ }^{\mathrm{c}}$ & $z_{f}^{\mathrm{d}}$ \\
\hline 631 & $8.48_{-0.34,-0.10}^{+0.24,+0.15}$ & $0.001_{-0.001,-0.001}^{+0.012,0.001}$ & $0.66_{-0.41,-0.09}^{+0.20,+0.14}$ & $0.00_{-0.00,-0.00}^{+0.01,+0.00}$ & $0.95_{-0.63,-0.03}^{+0.05,+0.05}$ & 4.0 \\
\hline 712 & $\begin{array}{l}8.00_{-0.48,-0.25}^{+0.46,+0.27} \\
\text {. }\end{array}$ & $\begin{array}{l}0.001_{-0.000,-0.000}^{+0.002,0.000}\end{array}$ & $0.45_{-0.42,-0.21}^{+0.37,+0.24}$ & $\begin{array}{l}0.02_{-0.02,-0.02}^{+0.02,+0.01} \\
\text { a. }\end{array}$ & $\begin{array}{l}0.41_{-0.40,-0.20}^{+0.31,+0.23} \\
\text { a }\end{array}$ & 5.2 \\
\hline 4442 & $6.90_{-0.18,-0.15}^{+0.29,+0.09}$ & $0.000_{-0.000,-0.000}^{+0.001,0.000}$ & $0.14_{-0.14,-0.14}^{+0.21,+0.05}$ & $0.04_{-0.04,-0.01}^{+0.01,+0.01}$ & $0.06_{-0.06,-0.06}^{+0.19,+0.04}$ & 5.8 \\
\hline 5183 & $7.13_{-0.06,-0.04}^{+0.08,+0.03}$ & $0.000_{-0.000,-0.000}^{+0.000,0.000}$ & $0.05_{-0.05,-0.05}^{+0.09,+0.02}$ & $0.05_{-0.01,-0.00}^{+0.00,+0.00}$ & $0.04_{-0.04,-0.04}^{+0.16,+0.04}$ & 4.8 \\
\hline 5225 & $8.57_{-0.45,-0.22}^{+0.40,+0.20}$ & $0.001_{-0.001,-0.001}^{+0.005,0.001}$ & $0.55_{-0.51,-0.15}^{+0.26,+0.17}$ & $0.03_{-0.02,-0.01}^{+0.02,+0.02}$ & $0.58_{-0.54,-0.21}^{+0.37,+0.32}$ & 5. \\
\hline 9040 & $8.33_{-0.18,-0.11}^{+0.19,+0.10}$ & $0.000_{-0.000,-0.000}^{+0.001,0.000}$ & $0.34_{-0.14,-0.08}^{+0.16,+0.08}$ & $0.00_{-0.00,-0.00}^{+0.03,+0.00}$ & $0.09_{-0.09,-0.09}^{+0.20,+0.05}$ & 4.9 \\
\hline 9340 & $\begin{array}{l}7.29_{-0.02,-0.01}^{+0.04,+0.01} \\
\text { a. }\end{array}$ & $0.000_{-0.000,-0.000}^{+0.001,0.000}$ & $0.01_{-0.01,-0.01}^{+0.02,+0.00}$ & $0.00_{-0.00,-0.00}^{+0.00,+0.00}$ & $\begin{array}{l}0.00_{-0.00,-0.00}^{+0.06,+0.01} \\
\text { a }\end{array}$ & 4.7 \\
\hline 9487 & $7.65_{-0.41,-0.26}^{+0.54,+0.21}$ & $\begin{array}{l}0.001_{-0.001,-0.001}^{+0.010,001}\end{array}$ & $0.26_{-0.26,-0.16}^{+0.31,+0.14}$ & $\begin{array}{l}0.02_{-0.02,-0.02}^{+0.02,+0.01} \\
\text { a. }\end{array}$ & $\begin{array}{l}0.47_{-0.46,-0.29}^{+0.43,+0.30} \\
\text { a }\end{array}$ & 4.1 \\
\hline L7-01 & $9.34_{-0.58,-0.36}^{+0.76,+0.38}$ & $0.001_{-0.001,-0.001}^{+0.087,0.003}$ & $1.41_{-1.18,-0.35}^{+0.60,+0.43}$ & $0.02_{-0.02,-0.02}^{+0.03,+0.01}$ & $0.00_{-0.00,-0.00}^{+0.70,+0.02}$ & 7.7 \\
\hline L8-01 & $8.55_{-0.90,-0.38}^{+0.84,+0.62}$ & $0.003_{-0.002,-0.002}^{+0.037,0.005}$ & $0.52_{-0.52,-0.51}^{+0.81,+0.24}$ & $0.03_{-0.02,-0.01}^{+0.02,+0.02}$ & $0.61_{-0.54,-0.20}^{+0.38,+0.39}$ & \\
\hline A383-iD1 & $\begin{array}{l}9.75_{-0.15,-0.08}^{+0.17,+0.08} \\
\text { a. }\end{array}$ & $\begin{array}{l}0.000_{-0.000,-0.000}^{+0.001,0.000} \\
\end{array}$ & $1.04_{-0.14,-0.08}^{+0.16,+0.07}$ & $0.00_{-0.00,-0.00}^{+0.01,+0.00}$ & $0.08_{-0.08,-0.08}^{+0.23,+0.06}$ & 6.0 \\
\hline CL0024-iD1 & $9.79_{-0.64,-0.30}^{+0.50,+0.40}$ & $\begin{array}{l}0.005_{-0.004,-0.004}^{+0.044,0.007} \\
\end{array}$ & $0.84_{-0.83,-0.48}^{+0.90,+0.48}$ & $0.02_{-0.02,-0.02}^{+0.02,+0.01}$ & $0.60_{-0.54,-0.20}^{+0.40,+0.40}$ & 6.8 \\
\hline CL0024-zD1 & $9.79_{-0.80,-0.39}^{+0.69,+0.45}$ & $0.004_{-0.004,-0.004}^{+0.069,0.007}$ & $1.25_{-1.19,-0.49}^{+0.87,+0.63}$ & $0.03_{-0.02,-0.01}^{+0.02,+0.02}$ & $0.54_{-0.49,-0.20}^{+0.46,+0.46}$ & \\
\hline
\end{tabular}

Notes. This table lists the median values for each parameter as well as the $95 \%$ and $68 \%$ credible regions, separated by a comma.

${ }^{a}$ Extinction (Calzetti et al. 2000).

${ }^{\mathrm{b}}$ Metallicity (Solar metallicity is $Z=0.02$ ).

${ }^{\mathrm{c}}$ Ionizing radiation escape fraction.

${ }^{\mathrm{d}}$ Earliest formation redshift. 


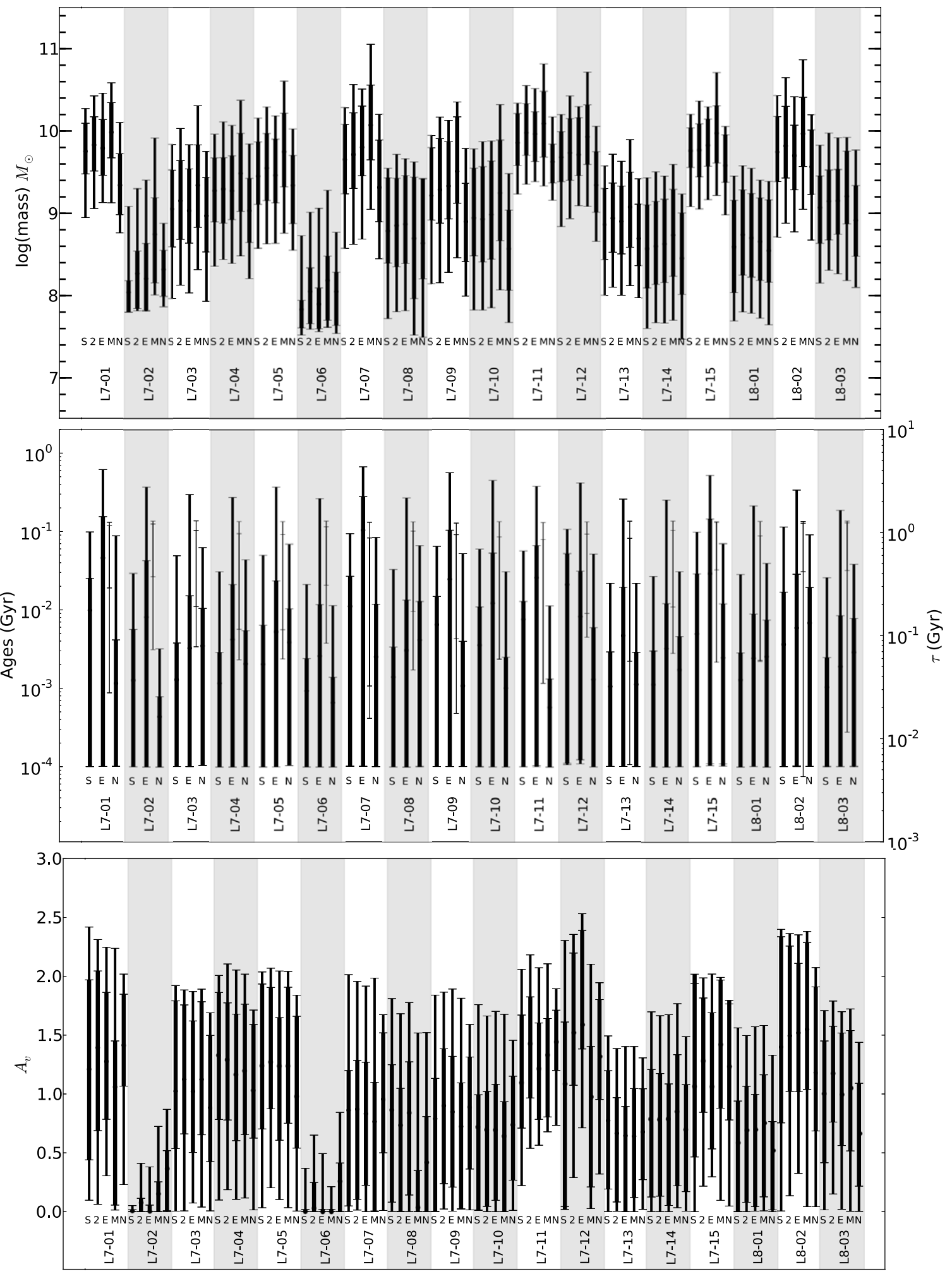

Figure 14. The 68\% and 95\% credible intervals for the individual sources listed in Labbé et al. (2010). The stellar mass is shown in the top panel. The stellar ages are shown in the middle panel. This panel also shows the value of $\tau$ next to the EXP model stellar ages. The extinction is shown in the bottom panel. The SSP, SSP2, EXP, Maximum $M / L$, and SSP+Nebular models are shown from left to right for each source and are labeled S, 2, E, M, and N, respectively.

to others with masses $>10^{10} M_{\odot}$ (e.g., compare L7-06 with L7-01 and L7-07).

Figure 14 shows the acceptable stellar ages also span a broad range of values. Although in every case we can exclude ages larger than 50-100 Myr old. This is in stark contrast to the results of Labbé et al. (2010), who claim ages of $300 \mathrm{Myr}$ and 700 Myr for the stacked $z \sim 7$ and $z \sim 8$ sources, respectively. The middle panel of Figure 14 shows the values of $\tau$ for the EXP model. It demonstrates that only moderate values $(<4 \mathrm{Gyr})$ are likely. Very large values of $\tau$ mean that the star formation history of these objects remains constant. This is again different from Labbé et al. (2010). There, the 

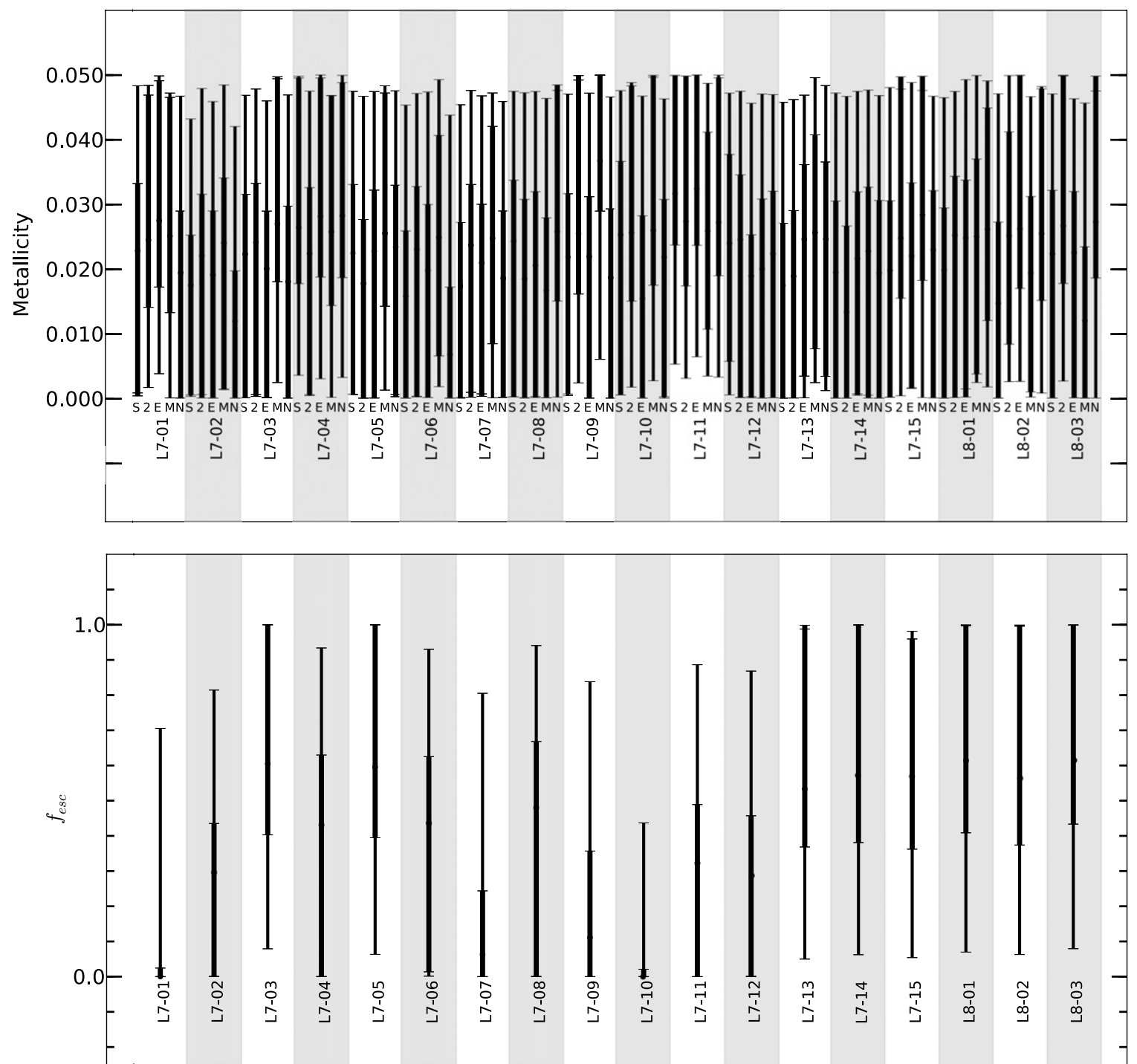

Figure 15. The $68 \%$ and $95 \%$ credible intervals for the individual sources listed in Labbé et al. (2010). The extinction, $A_{\mathrm{V}}$, is shown in the top panel. The nebular escape fractions are shown in the bottom panel.

authors assume a CSF rate for the models they fit to the observations.

The bottom panel of Figure 14 shows that the extinction varies greatly between sources. We see that a few of these sources (e.g., L7-01 and L7-06) are consistent with very low values of $A_{\mathrm{V}}$, while others have significantly larger values (e.g., L7-11, $A_{\mathrm{V}} \sim$ $1.5)$, and others have very large credible intervals, indicating we cannot constrain $A_{\mathrm{V}}$ well. This casts some doubts on the validity of deriving physical parameters from stacked images of these objects. Finally, Figure 15 shows that both metallicity and $f_{\text {esc }}$ are difficult to constrain. The latter is not surprising, given that at ages 50-100 Myr, the contribution from nebular emission should be negligible (e.g., Leitherer et al. 1999; Reines et al. 2010). Overall, we have demonstrated that the $\pi \mathrm{MC}^{2}$ analysis is able to provide robust information about the ages and masses of the individual objects. While some parameters are less reliably constrained, we are still able to derive useful information about the nature of these objects.

\subsubsection{Lensed $z>6$ Candidates}

We have applied the set of five models (SSP, SSP2, EXP, $\max M / L \mathrm{SSP} 2$, and SSP+Nebular) to six high-redshift candidates identified by Bradley et al. (2008), Zheng et al. (2009), and Richard et al. (2011). The objects are: A1689-zD1 from Bradley et al. (2008); A1703-iD1, A2218-iD1, CL0024-iD1, and CL0024-zD1 from Zheng et al. (2009); and A383-iD1 from Richard et al. (2011). All of these objects are reported to be galaxies amplified by gravitational lensing. These objects are interesting because they may provide important information on the nature of galaxies during the period of reionization. The lensing provided by massive foreground clusters amplifies their observed light and sizes, making it possible to not only detect these systems, but to also probe their properties. All three papers use standard $\chi^{2}$ fitting of $\mathrm{BC} 03$ templates to derive physical properties. Bradley et al. (2008) conclude that their object lies at $z \sim 7.6$ with a mass of $10^{9} M_{\odot}$ and a stellar population with an age of 45-300 Myr with no extinction. Zheng et al. (2009) find a mass of $\sim 10^{10} M_{\odot}$ for A1703-iD1 and $\sim 10^{9} M_{\odot}$ for CL0024-iD1 and CL0024-zD1 along with stellar ages of 40-80 Myr with no extinction for all three sources. They do not report results for A383-iD1. Finally, Richard et al. (2011) report that their object lies at $z \sim 6$ with a stellar mass of $\sim 10^{9}$, and no extinction. They derive a best-fit age of $\sim 800 \mathrm{Myr}$, which (as the authors note themselves) is difficult to reconcile given that the age of the universe at that redshift is $~ 900$ Myr. 


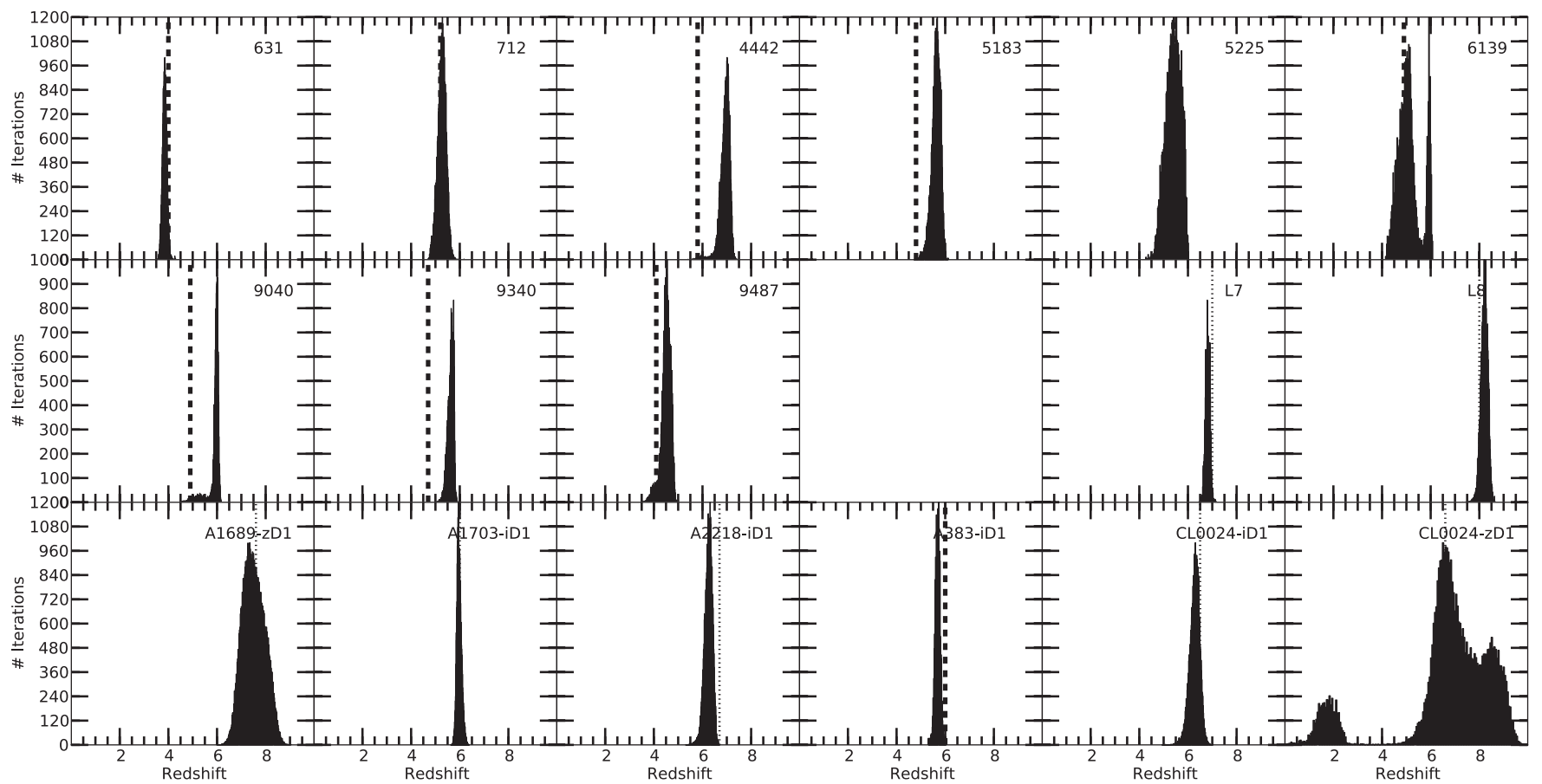

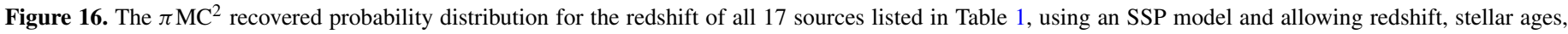
mass, and metallicity to vary. The spectroscopic redshifts are shown using thick dashed lines. Assumed photometric redshifts are shown using thin dotted lines.

Table 7

$\pi \mathrm{MC}^{2}$ SSP Results Using MA05 and BC03 Models for a Subsample of Sources from Table 1 and Allowing Only Stellar Mass, Stellar Ages, and Extinction to Vary

\begin{tabular}{|c|c|c|c|c|c|c|}
\hline \multirow[t]{2}{*}{ UID } & \multicolumn{3}{|c|}{ MA05 } & \multicolumn{3}{|c|}{$\mathrm{BC} 03$} \\
\hline & $\begin{array}{l}\text { Age } \\
\text { (Gyr) }\end{array}$ & $A_{\mathrm{V}}{ }^{\mathrm{a}}$ & $\begin{array}{l}\text { Mass } \\
\left(M_{\odot}\right)\end{array}$ & $\begin{array}{l}\text { Age } \\
\text { (Gyr) }\end{array}$ & $A_{\mathrm{V}}^{\mathrm{a}}$ & $\begin{array}{l}\text { Mass } \\
\left(M_{\odot}\right)\end{array}$ \\
\hline 4442 & $0.022_{-0.02}^{+0.05}$ & $0.22_{-0.22}^{+0.56}$ & $7.74_{-0.79}^{+0.61}$ & $0.045_{-0.05}^{0.11}$ & $0.28_{-0.28}^{0.54}$ & $8.20_{-0.66}^{0.57}$ \\
\hline 5183 & $0.013_{-0.01}^{+0.03}$ & $0.22_{-0.22}^{+0.56}$ & $7.86_{-0.57}^{+0.52}$ & $0.023_{-0.02}^{0.06}$ & $0.35_{-0.35}^{0.45}$ & $8.32_{-0.52}^{0.40}$ \\
\hline 5225 & $0.007_{-0.01}^{+0.01}$ & $0.58_{-0.32}^{+0.51}$ & $8.83_{-0.38}^{+0.35}$ & $0.016_{-0.02}^{0.01}$ & $0.73_{-0.38}^{0.36}$ & $9.44_{-0.35}^{0.22}$ \\
\hline 6139 & $0.002_{-0.00}^{+0.00}$ & $0.81_{-0.27}^{+0.23}$ & $8.95_{-0.34}^{+0.23}$ & $0.008_{-0.01}^{0.01}$ & $0.82_{-0.26}^{0.27}$ & $9.32_{-0.23}^{0.23}$ \\
\hline 9040 & $0.002_{-0.00}^{+0.01}$ & $0.65_{-0.54}^{+0.26}$ & $8.49_{-0.38}^{+0.34}$ & $0.009_{-0.01}^{0.01}$ & $0.64_{-0.29}^{0.30}$ & $8.91_{-0.30}^{0.28}$ \\
\hline 9340 & $0.017_{-0.02}^{+0.03}$ & $0.22_{-0.22}^{+0.61}$ & $8.08_{-0.58}^{+0.47}$ & $0.023_{-0.02}^{0.07}$ & $0.40_{-0.40}^{0.43}$ & $8.48_{-0.56}^{0.39}$ \\
\hline 9487 & $0.016_{-0.02}^{+0.02}$ & $0.17_{-0.17}^{+0.50}$ & $8.12_{-0.53}^{+0.36}$ & $0.036_{-0.04}^{0.03}$ & $0.20_{-0.20}^{0.46}$ & $8.66_{-0.34}^{0.21}$ \\
\hline
\end{tabular}

Notes. This table lists the median values for each parameter as well as the $95 \%$ credible regions.

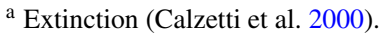

Figures 11 and 12 show the results when these lensed objects are analyzed using $\pi \mathrm{MC}^{2}$. All candidate objects except A383-iD1 show mass estimates that are $\approx 10^{10}$ regardless of the five models used. This is $\sim 2-5$ times larger than those obtained from the fits in Bradley et al. (2008) and Zheng et al. (2009) for A1703-iD1, CL0024-iD1, and CL0024-zD1, although it is consistent with the masses estimated for CL0024-iD1 and CL0024-zD1 (Zheng et al. 2009). A383-iD1 is discrepant as different models produce different results. This object could have a mass of $\sim 10^{9-10} M_{\odot}$ with a young population, low extinction, and high $f_{\text {esc }}$, or it could have a mass $>10^{11} M_{\odot}$ and a stellar age approaching the age of the universe (at that redshift). As a group, these lensed galaxies are significantly more massive than the GRAPES or Labbé et al. (2010) objects. We observe that the stellar age intervals, shown in Figure 11 for the SSP, EXP, and SSP+Nebular models, are very broad, as are the credible regions for $f_{\text {esc }}$ shown in Figure 12. This yields stellar age estimates for the six sources that are poorly constrained. Improved physical parameter estimates for these sources require significantly more accurate photometry. However, we show in Table 2 that the formation redshifts $\left(z_{\mathrm{f}}\right)$, based on the upper $95 \%$ credible intervals for the stellar age, are consistently less than $z=8.0$ for these sources.

\subsection{A Note about Template Models}

Briefly, we discuss possible differences between the stellar population models of $\mathrm{BC} 03$ and M05. It has been noted in the literature that these models may yield differences in derived stellar masses and ages (among other parameters), particularly at longer wavelengths (e.g., Maraston et al. 2006). We have tested possible differences using SSP models for a subsample of the GRAPES sources from Table 1. These tests compared the parameters of age, $A_{\mathrm{V}}$, and stellar mass. Table 7 shows the results of these comparisons. The only significant differences are in the derived ages. BC03 ages are $\sim 2$ times older than those from M05. The $A_{\mathrm{V}}$ and stellar masses are consistent with each other within the confidence intervals shown. It is likely that the reason these differences are less pronounced is that the comparisons between $\mathrm{BC} 03$ and $\mathrm{M} 05$ are at rest-frame optical wavelengths at these redshifts. At rest-frame wavelengths longer than $\sim 1 \mu \mathrm{m} \mathrm{BC03}$ and M05 show the greatest differences in parameters such as age, $A_{\mathrm{V}}$, and stellar mass.

We note that the M05 templates provide equally good fits to our observations and do not significantly alter the width of $95 \%$ credible regions we derive. The credible regions derived using the M05 models are therefore essentially identical in shape, but shifted slightly in age compared to those derived using BC03.

\subsection{High-redshift Photometric Redshifts}

As was mentioned in Section 1, redshift is one of the parameters that can be varied when applying $\pi \mathrm{MC}^{2}$, or any SED 


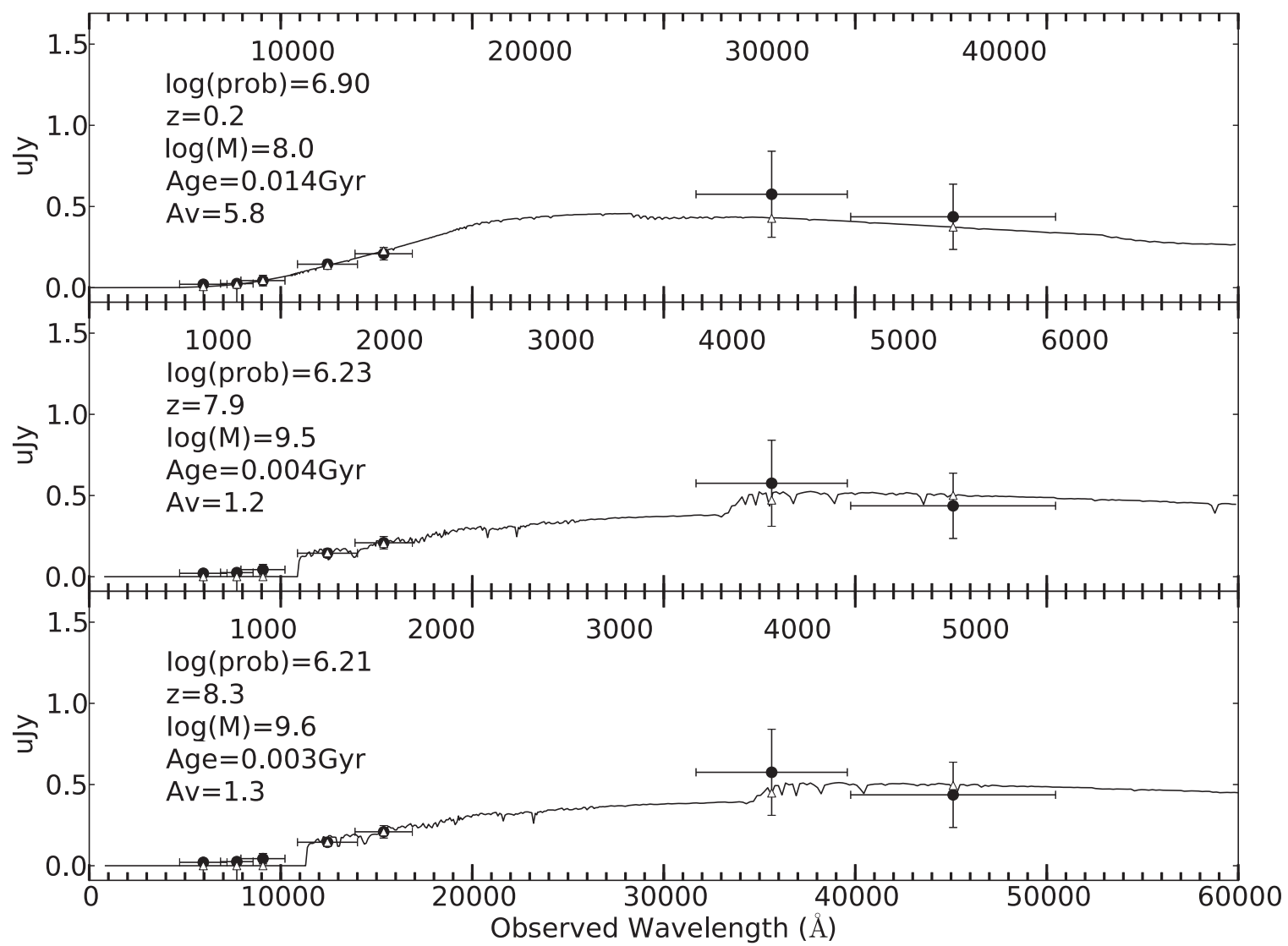

Figure 17. Possible solutions at $z<2.0,7.8<z<8.1$, and $z>8.1$ for object CL0024-zD1, shown in the top to bottom panels, respectively. Each panel shows the photometric redshift, derived stellar mass, age, and extinction, as well as the log-probability of the model that is overplotted. The rest-frame wavelength is listed at the top of each sub-plot. The black circles and associated error bars show the observations. The white triangle shows the model photometry in the same band passes. This illustrates the difficulty in ruling out that high-redshift sources, such as this one, a confirmed lensed object at redshift $\approx 6$, are not low-redshift interloper if one would only have access to broadband photometry.

fitting algorithm. The GRAPES sources were spectroscopically identified using strong Ly $\alpha$ emission. These identifications serve as the basis for the redshifts listed in Table 1. However, the nonGRAPES sources in Table 1 were photometrically selected. As an exercise, we applied $\pi \mathrm{MC}^{2}$ to all of the sources in Table 1, including the GRAPES sources, to see how well we could recover these claimed redshifts. The GRAPES sources serve as a control sample for this test.

The results are shown in Figure 16. The photometric redshifts derived for the GRAPES sample are relatively good matches to the spectroscopic ones. The probability distributions for the remaining objects are shown in Figure 16. Most redshifts are well defined. This is expected since the effect of the Lyman break is a very strong photometric feature. The lensed galaxy CL0024zD1, identified by Zheng et al. (2009) as a $z \sim 6-7$ galaxy, shows two possible solutions from the $\pi \mathrm{MC}^{2}$ analysis: $z>6$ or $z<$ 2.5. In Figure 17, we show SED solutions for CL0024-zD1 obtained at $z=0.2, z=7.9$, and $z=8.3$ with the photometric fluxes overplotted. These particular solutions are the best fits in the regions $z<2.0,7.8<z<8.1$, and $z>8$, respectively. This demonstrates how photometric redshifts can produce ambiguous results when using only a limited number of available filters. A larger number of observations, including deep $K$-band observations, deeper observations in the ACS bands (e.g., F606W, F775W, F850LP), and possibly low-resolution WFC3 spectra are needed to confirm that this source is not a lower redshift interloper.

\section{CONCLUSIONS AND FUTURE WORK}

We have introduced our implementation of a Markov Chain based, SED analysis package, $\pi \mathrm{MC}^{2}$. We have tested $\pi \mathrm{MC}^{2}$ extensively, first against simulated galaxies of known parameters, and then using real observations of putative high-redshift sources. This analysis has made it possible to ascertain the caveats of comparing observed high-redshift SEDs to models. Our main findings are as follows.

1. That a Bayesian based Monte Carlo Markov Chain analysis of the SEDs of high-redshift sources provides a robust picture of the nature of these sources. Unlike more traditional best-fit-based results, it can determine $95 \%$ credible intervals for each parameter, including which ones are degenerate and which ones remain largely unconstrained.

2. Stellar mass is the easiest parameter to constrain.

3. Photometric noise and choice of different star formation histories have an impact on derived stellar mass, age, metallicity, and extinction. In the case of stellar ages, it is not possible to estimate a minimum age for the sources, but it is possible to determine upper limits (i.e., objects are less than a certain age). Metallicity cannot be constrained with the current level of photometric errors. Extinction and nebular emission (via $f_{\text {esc }}$ ) are often not constrained. It is not uncommon for the uncertainty in $A_{\mathrm{V}}$ to be $>1.0$ while $f_{\text {esc }}$ can often take any values between 0 and 1 . Furthermore, some parameters, such as extinction and metallicity, can be biased, especially at higher redshifts. 
4. The addition of IRAC data, which probes the rest-frame optical light, is not enough to provide strong constraints for the stellar ages and extinction. Significantly improved photometric precision, on the order of $1 \%-2 \%$, is required to constrain these quantities.

5. We tested our ability to constrain physical properties of objects using several star formation histories (SSP, EXP, SSP2, Maximum $M / L$ SSP2) for $1<z<8$. We find that as redshift increases our ability to derive physical parameters decreases strongly.

6. We have applied our method, $\pi \mathrm{MC}^{2}$, to 33 sources at redshifts ranging from $4<z<8$ : nine GRAPES objects (Pirzkal et al. 2007), $z \sim 7-8$ objects (Labbé et al. 2010), and six lensed $z>6$ candidates (Bradley et al. 2008; Zheng et al. 2009; Richard et al. 2011). We find significant differences between $\pi \mathrm{MC}^{2}$ results and those in Labbé et al. (2010), particularly for stellar ages. We find for $2 / 4$ lensed galaxies from Zheng et al. (2009) mass differences in which $\pi \mathrm{MC}^{2}$ produces values $2 \times-5 \times$ larger. The other two are consistent with Zheng et al. (2009). In the case of the lensed galaxy from Bradley et al. (2008), we find three different credible redshift ranges, and two different, yet credible age and mass ranges (young and low mass or extremely old and massive). We also demonstrate for these sources that there is no statistically compelling evidence that any sources formed at a redshift larger than $z=8$.

We have shown that our implementation of $\pi \mathrm{MC}^{2}$ allows us to determine posterior credible intervals for the physical parameters of observed objects for a variety of input models, and with many possible parameters. Our tests showed that highprecision photometry is required to provide solid estimates of degenerate parameters such as age, extinction, and metallicity. The MCMC approach allows us to determine the range of possible model parameters in a manner that accounts for photometric errors as well as model degeneracies. Overall, we have found that the $\pi \mathrm{MC}^{2}$ approach to SED analysis is a powerful way to constrain physical parameters. It allows one to fit observations and derive statistically credible intervals even when presented with complex models, degenerate parameters, or "nuisance" parameters. Credible intervals are robust because they allow one to gauge the reliability (or believability) of each derived physical parameter. $\pi \mathrm{MC}^{2}$ deals with parameter degeneracies efficiently and allows us to easily identify the parameters that are the most important to the quality of the fit (e.g., Figure 5). This is an attractive alternative to simple, best-fit methods as the latter cannot make an assessment of the quality or reliability of each derived parameter, but rather yields a quality assessment of the entire fit. $\pi \mathrm{MC}^{2}$ and its Bayesian foundations let us identify a range of model parameter values that are consistent with the observations, assuming our model is correct. However, one must remain conscious of the fact that we cannot be certain that the models that are compared to our observations are appropriate. This is especially true at high redshifts where we have little evidence that stellar populations behave as they do in the local universe. Nevertheless, the exercises presented in this paper demonstrate that $\pi \mathrm{MC}^{2}$ is a robust tool for deriving information for high-redshift sources.
Finally, we note that $\pi \mathrm{MC}^{2}$ is not limited to broadband photometry alone. It can also be applied to low-resolution spectra ( $R \sim$ tens to 1000). Given the recent, successful deployment of the WFC3 grism during SMOV4, $\pi \mathrm{MC}^{2}$ is an attractive tool for analyzing such data. Furthermore, $\pi \mathrm{MC}^{2}$ is not limited to the high-redshift universe, and can be applied to the constraining physical parameters of (relatively) nearby galaxies using lowresolution spectra. Future work will include the analysis of optical and near-IR spectra of coalesced galaxy mergers, including luminous and ultraluminous infrared galaxies (e.g., Rothberg \& Fischer 2010).

We would like to thank the referee of this paper for constructive comments. This work is supported by Research Support Agreements (RSA) 1309908 at the Space Telescope Science Institute, which is operated by AURA under NASA contract NAS5-26555.

\section{REFERENCES}

Acquaviva, V., Gawiser, E., \& Guaita, L. 2011, ApJ, 737, 47

Beckwith, S. V. W., Stiavelli, M., Koekemoer, A., et al. 2006, AJ, 132, 1729

Bell, E. F., McIntosh, D. H., Katz, N., \& Weinberg, M. D. 2003, ApJS, 149, 289

Bernardo, J. M., Berger, J., Dawid, A. P., \& Smith, J. F. M. (ed.) 1992, Bayesian Statistics 4 (Oxford: Oxford Univ. Press)

Bradley, L. D., Bouwens, R. J., Ford, H. C., et al. 2008, ApJ, 678, 647

Bruzual, G., \& Charlot, S. 2003, MNRAS, 344, 1000

Calzetti, D., Armus, L., Bohlin, R. C., et al. 2000, ApJ, 533, 682

Dickinson, M., Papovich, C., Ferguson, C., \& Budava'ri, T. 2003, ApJ, 587, 25

Geweke, J. 1992, in Bayesian Statistics 4, ed. J. M. Bernardo et al. (Oxford: Oxford Univ. Press), 169

Inoue, A. K. 2011, MNRAS, 415, 2920

Labbé, I., Gonzlez, V., Bouwens, R. J., et al. 2010, ApJ, 716, 103

Lai, K., Huang, J.-S., Fazio, G., et al. 2007, ApJ, 655, 704

Leitherer, C., Schaerer, D., Goldader, J. D., et al. 1999, ApJS, 123, 3

Maraston, C. 2005, MNRAS, 362, 799

Maraston, C., Daddi, E., Renzini, A., et al. 2006, ApJ, 652, 85

McLure, R. J., Cirasuolo, M., Dunlop, J. S., Foucaud, S., \& Almaini, O. 2009, MNRAS, 395, 219

Mobasher, B., Dickinson, M., Ferguson, H. C., et al. 2005, ApJ, 635, 832

Nilsson, K. K., Møller, P., Möller, O., et al. 2007, A\&A, 471, 71

Nilsson, K. K., Östlin, G., Møller, P., et al. 2011, A\&A, 529, 9

Patil, A., Huard, D., \& Fonnesbeck, C. J. 2010, J. Stat. Softw., 35, 1

Pirzkal, N., Malhotra, S., Rhoads, J. E., \& Xu, C. 2007, ApJ, 667, 49

Pirzkal, N., Xu, C., Malhotra, S., et al. 2004, ApJS, 154, 501

Raftery, A. E., \& Lewis, S. M. 1995, in Practical Markov Chain Monte Carlo in Practice, ed. W. R. Gilks, S. Richardson, \& D. J. Spiegelhalter (London: Chapman and Hall), 115

Raiter, A., Fosbury, R. A. E., \& Teimoorinia, H. 2010, A\&A, 510, A109

Reines, A. E., Nidever, D. L., Whelan, D. G., \& Johnson, K. E. 2010, ApJ, 708, 26

Richard, J., Kneib, J.-P., Ebeling, H., et al. 2011, MNRAS, 414, L31

Rothberg, B., \& Fischer, J. 2010, ApJ, 712, 318

Sajina, A., Scott, D., Dennefeld, M., et al. 2006, MNRAS, 369, 939

Salpeter, E. E. 1955, ApJ, 121, 161

Schaerer, D., \& de Barros, S. 2009, A\&A, 502, 423

Schaerer, D., de Barros, S., \& Stark, D. P. 2011, A\&A, 536, A72

Serra, P., Amblard, A., Temi, P., et al. 2011, ApJ, 740, 22

Trotta, R. 2008, Contemp. Phys., 49, 71

Zackrisson, E., Bergvall, N., \& Leitet, E. 2008, ApJ, 676, L9

Zheng, W., Bradley, L. D., Bouwens, R. J., et al. 2009, ApJ, 697, 1907 